This item was submitted to Loughborough's Research Repository by the author.

Items in Figshare are protected by copyright, with all rights reserved, unless otherwise indicated.

\title{
Acoustic performance of a resonating perforated liner with incident axial and circumferential acoustic modes
}

PLEASE CITE THE PUBLISHED VERSION

https://doi.org/10.1016/j.jsv.2019.114897

\section{PUBLISHER}

Elsevier BV

VERSION

AM (Accepted Manuscript)

\section{PUBLISHER STATEMENT}

This paper was accepted for publication in the journal Journal of Sound and Vibration and the definitive published version is available at https://doi.org/10.1016/j.jsv.2019.114897

LICENCE

CC BY-NC-ND 4.0

\section{REPOSITORY RECORD}

Cassell, Mark, and Jon Carrotte. 2019. "Acoustic Performance of a Resonating Perforated Liner with Incident Axial and Circumferential Acoustic Modes”. figshare. https://hdl.handle.net/2134/9698909.v1. 


\title{
Acoustic performance of a resonating perforated liner with incident axial and circumferential acoustic modes
}

\author{
Mark A. Cassell*, Jon F. Carrotte \\ National Centre for Combustion and Aerothermal Technology (NCCAT), \\ Building 9, Holywell Park, Loughborough University, \\ Loughborough, LE11 3GR, United Kingdom
}

\begin{abstract}
Perforated liners are a common form of passive damping device used in engineering applications to damp acoustic pressure fluctuations. The liner has many orifices arranged over the surface with a rear cavity, where the liner can be designed to resonate akin to an array of Helmholtz resonators in parallel. However, whilst a Helmholtz resonator is insensitive to the incident mode, the large surface area and rear cavity of a perforated liner can generate internal mode shapes that affect the acoustic performance. This paper presents a quasi-one-dimensional analytical model capable of capturing the variation in acoustic performance as the internal cavity segmentation is altered with incident higher-order acoustic modes in a narrow annular duct. Thus, the model can allow the generation of circumferential mode shapes. The model shows, when the liner is highly segmented circumferentially, the liner behaviour is akin to that with an incident axial wave. The segmentation causes the internal cavity pressure to fluctuate uniformly at a similar frequency to a Helmholtz resonator with the same effective cavity dimensions. When the cavity length is significant relative to the wavelength, circumferential mode shapes are generated within the cavity and the frequency
\end{abstract}

(C) 2019. This accepted manuscript version is made available under the CC-BY-NC-ND 4.0 license http://creativecommons.org/licenses/by-nc-nd/4.0/.

This paper was accepted for publication in the Journal of Sound and Vibration and the definitive version is available at https://doi.org/10.1016/j.jsv.2019.114897. Please cite the published version.

${ }^{*}$ Corresponding author

Email address: m. cassell@lboro.ac.uk (Mark A. Cassell) 


\begin{tabular}{|c|c|c|c|}
\hline \multicolumn{4}{|c|}{ Nomenclature } \\
\hline$A$ & Cross-sectional area & $\eta$ & Compliance \\
\hline$c$ & Speed of sound & $\theta$ & Wall angle \\
\hline$f$ & Frequency & $\rho$ & Density \\
\hline$f_{c}$ & Cut-on frequency & $\sigma$ & Porosity \\
\hline$h$ & Stagnation enthalpy & $\varphi$ & Phase \\
\hline$k$ & Wave number & $\omega$ & Angular frequency \\
\hline$L$ & Length & & \\
\hline$m$ & Number of microphones & \multicolumn{2}{|c|}{ Mathematical symbols } \\
\hline$M$ & Mach number & $x^{\prime}$ & Time derivative of $x$ \\
\hline$n$ & Higher-order mode number & & \\
\hline$N_{S}$ & Number of sectors & \multicolumn{2}{|c|}{ Subscripts } \\
\hline$p$ & Pressure & 0 & Ambient condition \\
\hline$p_{0}$ & Total pressure & 1 & Damping skin parameter \\
\hline$P_{a}$ & Aperture pitch & 2 & Metering skin parameter \\
\hline$Q_{r}$ & Resonance parameter & $A$ & Incident clockwise wave \\
\hline$r$ & Radius & avg & Average \\
\hline$R$ & Reflection coefficient & $B$ & Incident anti-clockwise wave \\
\hline$r_{i}$ & Inner radius & $c$ & Cavity \\
\hline$r_{o}$ & Outer radius & $C$ & Reflected clockwise wave \\
\hline$r, \phi, z$ & Cylindrical coordinates & $d$ & Downstream value \\
\hline$S$ & Liner separation & $D$ & Reflected anti-clockwise wave \\
\hline$S t$ & Strouhal number & $i$ & Incident \\
\hline$t$ & Time & $r$ & Reflected \\
\hline$u$ & Velocity & $r, \phi, z$ & Cylindrical component \\
\hline$V$ & Volume & tot & Total \\
\hline$Z$ & Acoustic impedance & $z$ & Axial component \\
\hline$\Gamma$ & Inertia & $\phi$ & Circumferential component \\
\hline$\gamma$ & Specific heat ratio & \multirow{2}{*}{\multicolumn{2}{|c|}{ Superscripts }} \\
\hline$\delta$ & Admittance & & \\
\hline$\varepsilon$ & $\begin{array}{l}\text { Direction cosine of the wave- } \\
\text { front }\end{array}$ & $\begin{array}{l}+ \\
-\end{array}$ & $\begin{array}{l}\text { Upstream travelling wave } \\
\text { Downstream travelling wave }\end{array}$ \\
\hline
\end{tabular}


of resonance increases based on the circumferential frequency component. The model is then compared to an example experimental data set obtained from a facility designed to allow circumferential modes to cut-on simultaneously with an axial mode. A description of the facility and the multi-microphone decomposition method applied to decompose simultaneous modes of unknown orders and relative magnitudes is presented. The model has good agreement with the experimental results for a small cavity segmentation, although there is deviation observed at high frequencies when the cavity length becomes significant relative to the circumferential wavelength.

Keywords: passive damping device, higher-order acoustic mode, resonating perforated liner, linear wave superposition, reflection coefficient

\section{Introduction}

Passive damping devices are commonly used within engineering systems to damp acoustic pressure fluctuations. For small devices, such as Helmholtz resonators, spatial variations in the incident pressure wave are not typically an issue with the pressure variation across the neck inlet being negligible regardless of the mounting location. This is because the neck size is small relative to the acoustic wavelength. However, perforated liners, which consist of many distributed orifices on a plate, and may be backed by a cavity with a rigid rear wall or a second plate of distributed orifices, can have a large internal cavity and surface area. A non-uniform pressure distribution can occur across the face of the damper when the damper is:

1. fully annular or having a minimal number of separating walls in the cavity when a circumferential wave component is present; or

2. axially long.

This non-uniform pressure distribution can then lead to mode shapes forming within the internal cavity that affect the acoustic response of the damper. In this study, helical acoustic wave forms are considered in an infinitely narrow annular duct, which have both axial and circumferential wave components $\left(f_{\text {tot }}^{2} \approx f_{z}^{2}+f_{\phi}^{2}\right)$, in addition to plane axial waves. These waves will impinge upon a perforated liner mounted axially on the end of the annular duct such that the axial plane waves cause a uniform pressure fluctuation on the damper face.

Much work has been done to consider the behaviour of perforated liners with plane waves and the dependency of various geometric parameters. Generally 
however, the assumption is made that any variations in pressure across the damper surface are negligible and only mode shapes formed axially between the primary and secondary skin (or rear wall) are important. However, in the study by Eldredge and Dowling [1], the damper cavity is annular around the main cylindrical duct with no internal segmentation, with plane axial waves applied causing a significant axial variation across the skin and in the cavity. A double-skin system is used and it is shown that both liners are important for absorption. Troughs in the absorption occurred when pressure nodes were present in the cavity as the axial length became significant. Passive damping devices may be employed in a variety of applications including automotive exhaust or gas turbine combustion systems. In these environments the damper may be subject to high temperatures that could cause hot gas ingestion and detune the damper. Thus, a bias flow may be required for cooling or purging purposes. The bias flow rate has been studied in several cases $[2,3,4]$ alongside dependency studies on the cavity volume and skin porosities; generally, an increasing bias flow causes a decrease in the peak absorption and broadening of the response. However, Eldredge and Dowling [1] (and Macquisten et al. [5] for high temperature validation) found the bias flow capable of improving the effectiveness of the liner as it aids the dissipation of vortices generated from the aperture edges.

In some circumstances, it can be assumed that the perforated liner cavity acts akin to a Helmholtz resonator with the internal cavity volume divided between the damping skin apertures. The large pressure fluctuations within the cavity at resonance can cause flow reversal across the primary skin, which can necessitate the use of a purging bias flow to prevent hot gas ingestion. For this resonant case, the secondary skin, is used to meter the flow with a large pressure drop relative to the primary damping skin. In addition the apertures within the metering skin have a high length-to-diameter ratio. Thus, they have high acoustic inertias and therefore the skin has a minimal acoustic response allowing the cavity to resonate [2]. To effectively resonate, the cavity dimensions and the spacing between apertures are presumed to be insignificant compared to the acoustic wavelength such that they fill instantaneously creating a uniform isentropic fluctuation throughout the damper cavity. However, with a non-uniform incident pressure the resonance of the liner may be affected.

Annular or cylindrical duct geometries may be employed within practical engineering environments, for example within a gas turbine combustor, in which circumferential modes can exist. If the damper is mounted around the azimuth, circumferential pressure variations may occur; although, axial variations may still be ignored if the dimension is kept small relative to the axial wavelength. Eldredge 
[6] develops a model to consider a fully annular cavity, which is subjected to circumferential modes and finds that the results strongly agree with those obtained from the plane wave model developed by Eldredge and Dowling [1]. Modelling the effect of segmentation within the cavity with significant pressure variations on the damping skin, which can lead to internal mode shapes, however have not been widely considered. Rupp [3] created an initial one-dimensional model, based on the work by Eldredge and Dowling [1], where the wave was treated as a plane wave and the damper length assumed to be comparable to the excitation wavelength. This creates pressure variations along the damper surface and causes the onset of mode shapes in the cavity, leading to a significant decrease in energy loss as the length of the damper increases.

In a real world engineering application the structure of the incident acoustic mode can vary substantially in time with different acoustic modes existing simultaneously. Bauerheim et al. [7] experimentally show this variation with multiple modal structures coexisting in gas turbine combustors and instabilities that switch successively between dominantly standing and spinning. These experimental studies on higher-order acoustic modes within combustion chambers to analyse modal structures are rare, due to the complexity and cost involved. Large-eddy simulations may be used to analyse these structures, as in the study by Wolf et al. $[8,9]$; although, the computational resources required mean such studies are generally prohibitive. For example, in the case by Wolf et al. [8], 400000 CPU hours were required for 0.1 seconds of physical time. However, these experimental and computational investigations showed that the geometry of the combustor can influence the mode that dominates, and whether the entire system couples through the cooling tiles in the walls of the combustor to the feed annuli [10]. The presence of asymmetry can force the domination of a circumferentially standing mode. Modal coupling can be explained with a simple example. Evesque and Polifke [11] assume that in an annular system with a single point of asymmetry, for example, a single blocked section of an annular damper, the resulting acoustic pressure field must be asymmetric because of this circumferential variation. Therefore, if a purely axial (and axisymmetric) mode is incident upon this damper it must cause a superposition of circumferential modes to describe this asymmetric acoustic field with a scattering of energy to acoustic modes of various orders. Additionally, the scattered energy from this pure axial mode would lead to the formation of a circumferentially standing acoustic field because of the fixed point of asymmetry.

Parrott and Watson [12] and Rademaker et al. [13] experimentally investigate the effects of both circumferential and radial higher-order modes in a circular duct and the absorption provided by a circumferentially segmented liner. Two 
sets of microphones are utilised, one set upstream of the liner to control the amplitude and phase of nine loudspeakers, and the other set of microphones positioned downstream of the liner to calculate the absorption before an anechoic termination. Parrott and Watson [12] found that the desired dominant incident mode contained more than $95 \%$ of the total energy with other incident modes from the sources being suppressed. Using this method, the modal scattering, or the redistribution of acoustic energy to modes of different orders, could be measured and evaluated as caused by asymmetry of the liner. The downstream microphones are traversed radially and circumferentially during operation to enable decomposition of the field. However, limitations on the radial distance were applied as in the centre of the duct strong acoustic pressure gradients occur, which make the field sensitive to the presence of the microphone rake [13]. Gerhold et al. $[14,15]$ utilise a similar technique to analyse the impact of higher-order modes and modal scattering in a rectangular duct. Modal scattering causes energy to scatter equally between higher- and lower-order mode numbers, and the use of a segmented liner with varying impedance can provide more effective broadband performance and an equivalent peak absorption [16, 17, 18]. However, as Campos and Oliveira [19] note while redistribution of energy may increase the apparent absorption of a single mode, it can reinforce other modes that are potentially more difficult to damp. Tester et al. [20] show that lower-order modes are generally more difficult to absorb due to the relatively longer wavelengths associated with the modes. Rigid mechanical joins and splices are used to fix and separate adjacent liner panels circumferentially and/or axially. The splices can make the damper face smaller relative to the wavelength and may thus cause a significant reduction in acoustic performance at some conditions. The redistribution of this energy from these rigid splices can be shown computationally to be a function of the number and widths of the splices; although, the precise mechanics are poorly understood with the three-dimensional analysis being computationally demanding [18, 20, 21, 22, 23, 24].

It is necessary to consider these mixed acoustic mode structures and their impact on perforated liners; although, while the acoustic field is linear and uncoupled, as is assumed here, each mode may be considered independently. If the acoustic field is non-linear, and becomes coupled, then it cannot be described according to the theory of linear superposition. This theory relies on the calculation of the amplitudes of each mode by balancing the excitation and damping forces, and is thus potentially unverified for the non-linear case [25]. The non-linear effects can include frequency spreading, non-linear wave interaction (as opposed to the linear superposition) and non-linear absorption. For most practical damping 
applications, the wave is assumed to remain linear away from the face of the damper and henceforth adequately described by the linear wave equations, with the total field following the linear superposition of waves [26]. Additionally, any fluctuations in the density due to the pressure perturbations are small and linearised theory may thus be initially applied. Henceforth, non-linear acoustics shall not be considered here, and the field is assumed to remain uncoupled, although asymmetric segmentation of the damper could couple the system. By assuming the response remains uncoupled this simplifies the modelling process and allows for the development of an initial modelling tool. The model detailed here enables the prediction of a perforated damping liner that can have incident axial and circumferential modes. To this end the influence of various parameters on the damper can be investigated along with characterisation of the potential mode shape within the cavity. These could then be used to optimise the response of the damper, with respect to the frequency and magnitude of peak loss, when in the presence of both axial and circumferential modes. Furthermore, it is the purpose of this study to experimentally investigate the variation in performance of a perforated liner as the segmentation of the cavity is altered. This experimental data can be used to generate test cases to validate this and other models. The results that will be presented here, from the larger experimental data set, are concentrating on the first-order circumferential mode with an incident dominantly circumferentially standing acoustic field and segmentation that is applied axisymmetrically as far as possible. This will minimise any potential errors and interference arising from acoustic modal coupling from the duct terminations. The field is dominantly circumferentially standing with an average relative amplitude of 1.04 between the circumferentially opposing incident waves.

\section{Analytical model}

The model developed by Bellucci et al. [27, 28] can be used to model the absorption of a perforated liner damper or Helmholtz resonator across both the linear and non-linear absorption regimes. It assumes that an incident acoustic pressure wave will create a uniform pressure fluctuation throughout the cavity; hence, it can be used to model a damper mounted normal to the direction of propagation of an axial wave in an annular duct (e.g. it is mounted on the end of the duct). Thus, any circumferential cavity segmentation (assuming a constant cavity volume) may be disregarded. It is also assumed that when modelling the perforated liner the apertures have a sufficient pitch-to-diameter ratio such that they can be considered independently. This model is hereafter referred to as the 'axial wave model'. 


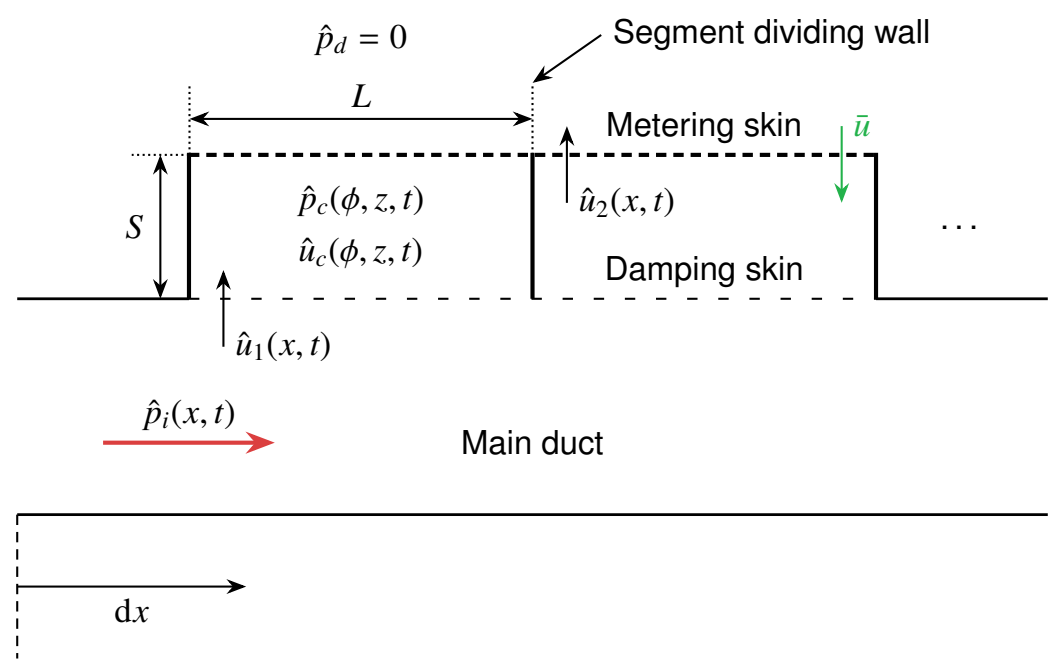

Figure 1: Perforated axial liner geometry as used by Rupp [3] for modelling a segmented damper system

However, with a significant variation across the damper surface or in the damper cavity this axial wave model will become invalid. Eldredge and Dowling [1] developed a one-dimensional model to investigate the absorption of axial plane waves, below the cut-on of higher-order modes, by a fully annular liner that is mounted parallel to the direction of propagation of the waves. Thus, the axial length of the liner can be significant. Eldredge [6] extended the model to analyse the impact of circumferential modes in a narrow annular duct and the resulting three-dimensional interaction using a Green's function approach. It was found, however, that the one-dimensional approach remained valid at frequencies above cut-on. Similar behaviours are noted between the modes when plotted as a function of the axial frequency component. Rupp [3] adapts the model by Eldredge and Dowling [1] to approximate a segmented damper system with a pure spinning wave in a duct of infinite radius, as illustrated in Fig. 1. It equates the system to an axially travelling wave that runs parallel to the liner and thus creates a pressure distribution along the axial dimension of the damping skin. It demonstrates the potential importance of the number of cavity divisions going between many small cavities, which could resonate, to a single cavity that can form mode shapes. As the cavity length increases, this causes the absorption predicted by the model to sharply decrease. Practical considerations, however, mean that how the cavity is segmented may not be purely down to acoustic damping reasons.

Here the described quasi-one-dimensional model is intended to assess the 


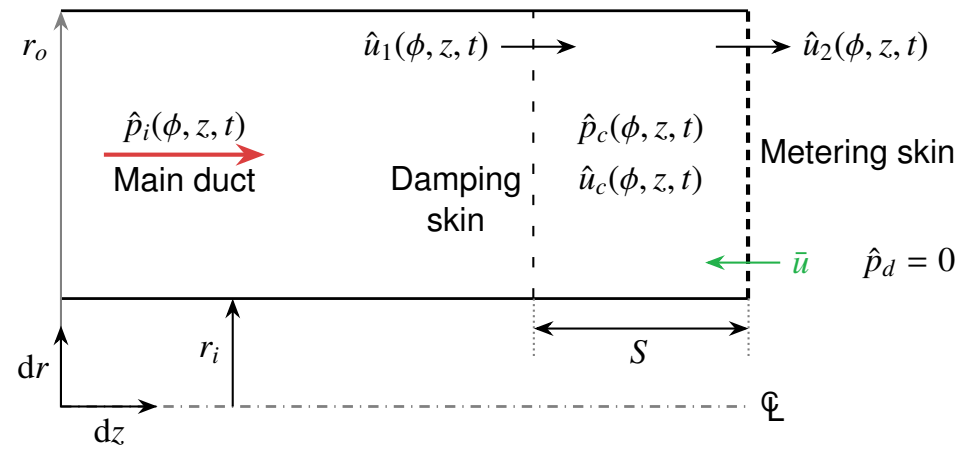

Figure 2: Axial cross-sectional view of the perforated liner mounted on the end of an annular main duct in the helical wave model.

impact of circumferential modes that may travel helically with circumferential and axial wave components. The damper is mounted normal to the direction of propagation of the axial mode at the duct termination (as illustrated in Fig. 2), with the duct and the damper having the same annular diameters and thus having consistent circumferential cut-on frequencies. A double perforated skin is presented here, where the model follows a similar method to that by Eldredge and Dowling [1] and Rupp [3]. This model is hereafter referred to as the 'helical wave model'. The acoustic velocity amplitude variation with the cavity $\left(\hat{u}_{c}\right)$ is characterised as:

$$
\frac{\partial \hat{u}_{c}(\phi, z, t)}{r_{c} \partial \phi}=-\frac{\mathrm{i} \hat{h}_{c}(\phi, z, t) \omega}{c^{2}}+\frac{\hat{u}_{1}(\phi, z, t)}{S}-\frac{\hat{u}_{2}(\phi, z, t)}{S}
$$

where the term $r_{c}$ is the mean radius of the damper cavity, $\theta$ is the sector angle ( $2 \pi$ for the fully annular case). The change in stagnation enthalpy within the cavity $\left(\hat{h}_{c}\right)$ is defined as:

$$
\frac{\partial \hat{h}_{c}(\phi, z, t)}{r_{c} \partial \phi}=-\frac{\mathrm{i} \omega \hat{u}_{c}(\phi, z, t)}{\varepsilon_{\phi}}
$$

where the term $\varepsilon_{\phi}$ is the direction cosine of the circumferential mode wave-front relative to the circumferential axis.

The fluctuating flow across the damping (primary) and metering (secondary) skins is related to the stagnation enthalpy across the liners and each skin compliance:

$$
\begin{aligned}
& \hat{u}_{1}(\phi, z, t)=\frac{\varepsilon_{\phi} \eta_{1}}{\mathrm{i} \theta \omega S}\left(\hat{h}_{c}(\phi, z, t)-\hat{h}_{0}(\phi, z, t)\right) \\
& \hat{u}_{2}(\phi, z, t)=\frac{\varepsilon_{\phi} \eta_{2}}{\mathrm{i} \theta \omega S}\left(\hat{h}_{d}(\phi, z, t)-\hat{h}_{c}(\phi, z, t)\right)
\end{aligned}
$$




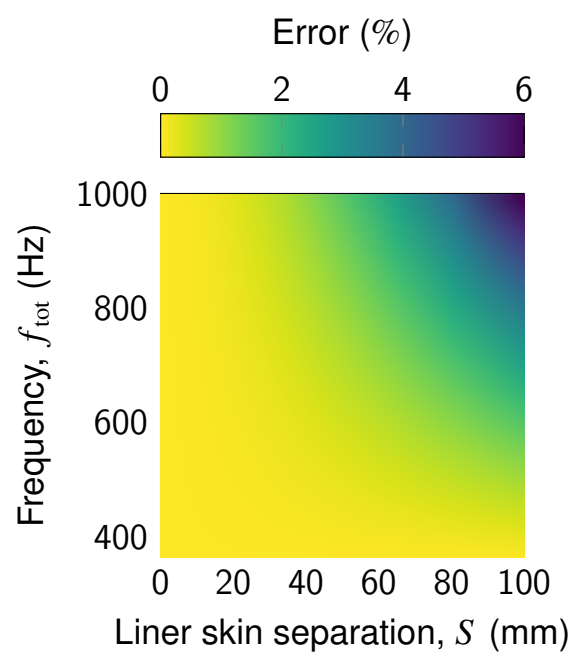

(a)

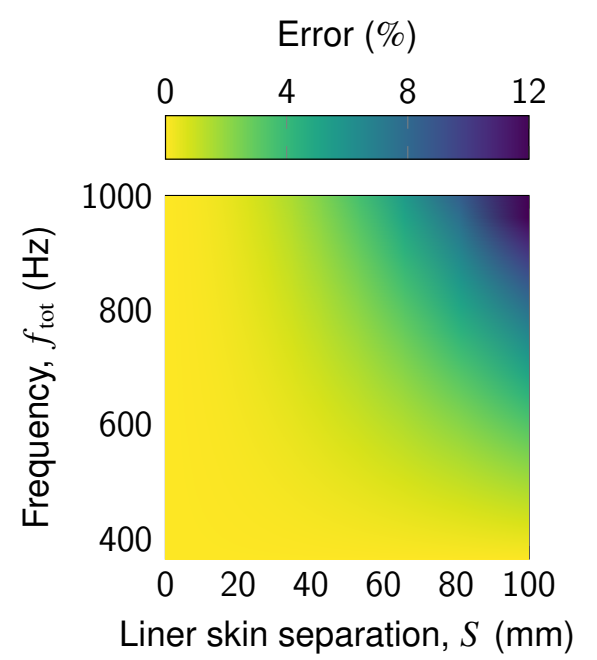

(b)

Figure 3: Sensitivity of the model to axial length of the cavity and frequency based on the percentage error between the left- and right-hand sides of the associated equations evaluated for an acoustic helical wave propagating without loss. (a) Eq. 1 and (b) Eqs. 3 and 4.

The model is regarded as being quasi-one-dimensional as the axial length of the cavity is considered to be negligible relative to the wavelength. Hence, only the circumferential variation is considered within the cavity. However, to estimate the errors associated with this assumption an acoustic wave can be considered that propagates, without loss, along the narrow annular duct. For this configuration the acoustic wave pressure (and be association the wave velocity and enthalpy) at axial positions separated by a distance $S$ can be considered. Using Eq. 1 the imbalance between the left- and right-hand sides can be evaluated at $z=0$ (for $\hat{u}_{1}$ ), $z=S / 2$ (for $\hat{u}_{c}, \hat{h}_{c}$ ), and $z=S$ (for $\hat{u}_{2}$ ), this imbalance being due to the left-hand side of the equation ignoring any velocity variation in the axial direction. Figure 3 shows the associated error which is, not surprisingly, sensitive to both frequency and separation distance $(S)$. However, at $1000 \mathrm{~Hz}$ a cavity depth greater than $40 \mathrm{~mm}$ is required for $1 \%$ error. Similarly, errors associated with differences in the left- and right-hand sides of equations Eq. 3 or 4 can also be evaluated (both equations produce comparable results), in which a porosity of $100 \%$ is assumed for calculation of the liner compliance. In this case a cavity depth greater than $40 \mathrm{~mm}$ is required to give an error greater than $2 \%$ at $1000 \mathrm{~Hz}$. The system that is experimentally investigated in this paper has a liner skin separation of $22.3 \mathrm{~mm}$. Hence, over the range of frequencies investigated (up to $1000 \mathrm{~Hz}$ ) it is assumed the errors associated with ignoring the axial variation in enthalpy and velocity 
within the cavity are acceptable.

The compliance for each skin is [3]:

$$
\eta=\frac{2 \theta \sigma r_{c}(\Gamma(S t)+\mathrm{i} \delta(S t))}{\pi r}
$$

The inertia $(\Gamma)$ and admittance $(\delta)$, which are functions of the Strouhal number (here using the definition of $S t=\omega r / \bar{u}$ ), can be related to the axial impedance of each skin by:

$$
\Gamma(S t)+\mathrm{i} \delta(S t)=-\frac{\mathrm{i} \pi \rho \omega r}{2} \frac{1}{Z_{z}}
$$

where the axial impedance here is calculated from the unsteady momentum balance across each skin given by Bellucci et al. [27]. It is capable of modelling both the linear and non-linear absorption regimes based on the ratio between the bias flow and acoustic velocities. When the bias flow is greater than the acoustic velocity absorption occurs within the linear regime wherein absorption is independent of the incident pressure amplitude. However, in the non-linear absorption regime, caused by a relatively small or no bias flow, the amount of energy absorbed is a function of the incident acoustic pressure with diminishing returns as the pressure increases.

While the bias flow velocity through the orifices may be significant, in the cavity and upstream duct this velocity component is negligible under all normal operating conditions. Thus, the fluctuating stagnation enthalpy is simplified as $\bar{u}_{c}=\bar{u}_{0}=0$, such that it is defined as $\hat{h}=\hat{p} / \bar{\rho}[1]$, and hence the damping skin velocity fluctuation given by Eq. 3 becomes:

$$
\hat{u}_{1}(\phi, z, t)=\frac{\varepsilon_{\phi} \eta_{1} c^{2}}{\mathrm{i} \gamma \theta \omega S}\left(\frac{\hat{p}_{c}(\phi, z, t)}{\bar{p}_{c}}-\frac{\hat{p}_{i}(\phi, z, t)}{\bar{p}_{0}}\right)
$$

For the metering skin, with the assumption that upstream of the metering skin is a plenum, so $\hat{h}_{d}(\phi, z, t)=0$ and thus Eq. 4 becomes:

$$
\begin{aligned}
\hat{u}_{2}(\phi, z, t) & =\frac{\varepsilon_{\phi} \eta_{2}}{\mathrm{i} \theta \omega S}\left(-\hat{h}_{c}(\phi, z, t)\right) \\
& =\frac{\varepsilon_{\phi} \eta_{2} c^{2}}{\mathrm{i} \gamma \theta \omega S}\left(-\frac{\hat{p}_{c}(\phi, z, t)}{\bar{p}_{c}}\right)
\end{aligned}
$$

The acoustic velocity in the cavity can be related to the circumferential pressure variation, per Eq. 2:

$$
\hat{u}_{c}=-\frac{\varepsilon_{\phi}}{\mathrm{i} \rho \omega r_{c}} \frac{\partial \hat{p}_{c}}{\partial \phi}
$$


The incident travelling acoustic wave in the narrow annular duct is based upon the clockwise $\left(\hat{p}_{A}\right)$ and anti-clockwise $\left(\hat{p}_{B}\right)$ rotating incident pressure amplitudes for the circumferential mode:

$$
\hat{p}_{i}(\phi, z, t)=\hat{p}_{A} \mathrm{e}^{\mathrm{i}\left(\omega t-\phi n-k_{z} z\right)}+\hat{p}_{B} \mathrm{e}^{\mathrm{i}\left(\omega t+\phi n-k_{z} z\right)}
$$

The model remains valid, and yields only negligible differences for all relative combinations of incident pressure amplitudes, so for example where $0 \leq \hat{p}_{A} \leq$ $\hat{p}_{B}$. A circumferentially standing wave has relative pressure amplitudes that are approximately equal $\left(\hat{p}_{A} \approx \hat{p}_{B}\right)$. A purely circumferentially spinning wave requires one incident component to be approximately zero $\left(\hat{p}_{A} \approx 0\right.$ or $\left.\hat{p}_{B} \approx 0\right)$.

By substitution and differentiating with respect to the circumferential coordinate it is possible to obtain an inhomogeneous ordinary differential equation of the second-order with respect to the circumferential component:

$$
\frac{\partial^{2} \hat{u}_{c}(\phi, z, t)}{\partial \phi^{2}}-\alpha \hat{u}_{c}(\phi, z, t)=\beta\left(\hat{p}_{A} \mathrm{e}^{\mathrm{i}\left(\omega t-\phi n-k_{z} z\right)}-\hat{p}_{B} \mathrm{e}^{\mathrm{i}\left(\omega t+\phi n-k_{z} z\right)}\right)
$$

where the constants $\alpha$ and $\beta$ are independent of space and given here by:

$$
\begin{gathered}
\alpha=\left(\frac{-\mathrm{i} \theta \omega^{2} S^{2}+\mathrm{i} \varepsilon_{\phi} c^{2}\left(\eta_{1}+\eta_{2}\right)}{\gamma \theta \omega \bar{p}_{c} S^{2}}\right)\left(-\frac{\mathrm{i} \rho \omega r_{c}}{\varepsilon_{\phi}}\right) \\
\beta=\left(\frac{\varepsilon_{\phi} \eta_{1} c^{2} r_{c}}{\mathrm{i} \gamma \theta \omega \bar{p}_{0} S^{2}}\right)(-\mathrm{i} n)
\end{gathered}
$$

Solving Eq. 12 gives the general solution as:

$u_{c}(\phi, z, t)=\operatorname{Const}_{1}(z, t) \mathrm{e}^{-\phi \sqrt{\alpha}}+\operatorname{Const}_{2}(z, t) \mathrm{e}^{\phi \sqrt{\alpha}}-\frac{\beta\left(\hat{p}_{A} \mathrm{e}^{\mathrm{i}\left(\omega t-\phi n-k_{z} z\right)}-\hat{p}_{B} \mathrm{e}^{\mathrm{i}\left(\omega t+\phi n-k_{z} z\right)}\right)}{\alpha+n^{2}}$

where the unknown constants Const $1(z, t)$ and Const $_{2}(z, t)$ are independent of the circumferential coordinate and can be solved by defining the relevant boundary conditions. In the case where the cavity is circumferentially divided into sectors, the circumferential velocity amplitude at the dividing walls must be a node:

$$
\hat{u}_{c}(\phi=0, z, t)=\hat{u}_{c}(\phi=\theta, z, t)=0
$$

Alternatively, for a fully annular cavity the boundary conditions must ensure the acoustic velocity remains periodic throughout the cavity such that:

$$
\begin{aligned}
\hat{u}_{c}(\phi=0, z, t) & =\hat{u}_{c}(\phi=\theta=2 \pi, z, t) \\
\frac{\mathrm{d} \hat{u}_{c}(\phi=0, z, t)}{\mathrm{d} \phi} & =\frac{\mathrm{d} \hat{u}_{c}(\phi=\theta=2 \pi, z, t)}{\mathrm{d} \phi}
\end{aligned}
$$


Thus producing:

$$
\begin{gathered}
\operatorname{Const}_{1}(z, t)=\frac{\beta\left(\mathrm{e}^{\mathrm{i}\left(\omega t-k_{z} z\right)} \mathrm{e}^{\phi \sqrt{\alpha}}\left(\hat{p}_{A}+\hat{p}_{B}\right)-\hat{p}_{A} \mathrm{e}^{\mathrm{i}\left(\omega t-\phi n-k_{z} z\right)}-\hat{p}_{B} \mathrm{e}^{\mathrm{i}\left(\omega t+\phi n-k_{z} z\right)}\right)}{\left(\alpha+n^{2}\right)\left(\mathrm{e}^{\phi \sqrt{\alpha}}-\mathrm{e}^{-\phi \sqrt{\alpha}}\right)} \\
\operatorname{Const}_{2}(z, t)=\frac{\beta \mathrm{e}^{\mathrm{i}\left(\omega t-k_{z} z\right)}}{\alpha+n^{2}}-\operatorname{Const}_{1}(z, t)
\end{gathered}
$$

The acoustic pressure in the cavity can hence be calculated and used to solve for the acoustic velocity amplitudes over the skins. These acoustic velocities can then be used to calculate the skin axial impedances $\left(Z_{z}=\hat{p} / \hat{u}_{z}\right)$ and yield a total representative axial impedance boundary condition for each mode. The total acoustic pressure and axial velocity fluctuations are based on the incident and reflected components such that:

$$
\begin{gathered}
\hat{p}(\phi, z, t)=\hat{p}_{i}(\phi, z, t)+\hat{p}_{r}(\phi, z, t) \\
\hat{u}_{z}(\phi, z, t)=\frac{\varepsilon_{z} \hat{p}_{i}(\phi, z, t)}{\rho c}-\frac{\varepsilon_{z} \hat{p}_{r}(\phi, z, t)}{\rho c}
\end{gathered}
$$

where the term $\varepsilon_{z}$, as defined by Morfey [29], is the direction cosine of the circumferential mode wave-front relative to the axial axis. Thus, for an axial wave $\varepsilon_{z}=1$. If the duct mean flow is sufficiently small such that $M \approx 0$, as in the current investigation where the Mach numbers in the duct and cavity are less than $M=0.002$, then the wave-front direction cosine can be calculated using:

$$
\varepsilon_{z}=\sqrt{1-\frac{k^{2}-k_{z}^{2}}{k^{2}}}
$$

Using Eqs. 21 and 22 the axial impedance can hence be written using the reflection coefficient $\left(R=\hat{p}_{r} / \hat{p}_{i}\right)$ :

$$
\begin{aligned}
Z_{z} & =\frac{\rho c\left(\hat{p}_{i}+\hat{p}_{r}\right)}{\varepsilon_{z}\left(\hat{p}_{i}-\hat{p}_{r}\right)} \\
& =\frac{\rho c(1+R)}{\varepsilon_{z}(1-R)}
\end{aligned}
$$

Thus, the axial impedance can be used to determine the reflection coefficient:

$$
R=\frac{\varepsilon_{z} Z_{z}-\rho c}{\varepsilon_{z} Z_{z}+\rho c}
$$

Results from the helical wave based model are shown in Fig. 4 with only 


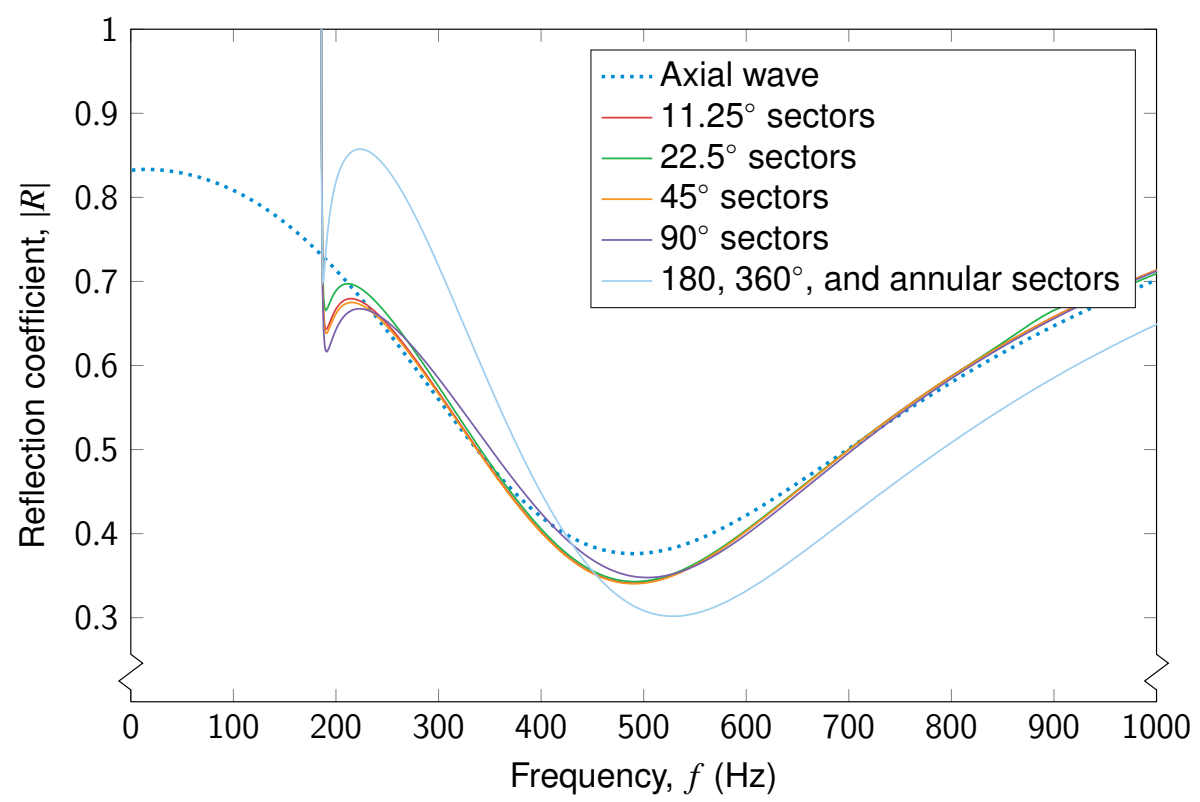

Figure 4: Effect of segmentation using the helical wave model for an incident first-order mode on the total reflection coefficient of the damper.

first-order helical waves and axial waves considered. By segmenting the cavity into sectors with walls, seven configurations are evaluated, with the acoustic response of the complete system considered for a direct comparison. In addition to the annular cavity that has no walls, there is a $360^{\circ}$ sector with one dividing wall, $2 \times 180^{\circ}$ sectors, $4 \times 90^{\circ}$ sectors, $8 \times 45^{\circ}$ sectors, $16 \times 22.5^{\circ}$ sectors, and $32 \times$ $11.25^{\circ}$ sectors. The configurations assume the cavity is axisymmetrically divided with a constant total skin porosity. The axial wave model predicts a constant response for the different configurations as it is assumed the dividing walls are infinitely thin and therefore the combined cavity volume remains constant. Due to the infinitely thin wall assumption it calculates the $180,360^{\circ}$, and annular sectors as all having a consistent response. While the cavity is highly segmented (e.g. $22.5^{\circ}$ sectors), with the angular length of the cavity being small relative to the wavelength of the circumferential component, the cavity resonates without significant circumferential variation as with an incident axial wave. Although when the cavity is relatively large, for example with a single dividing cavity wall $\left(360^{\circ}\right)$ or annular, mode shapes can form within the cavity. This causes the frequency at which peak absorption occurs to increase and the minimum reflection coefficient to decrease. The frequency increase relates to the axial and circumferential frequency components within the cavity, with the total frequency 


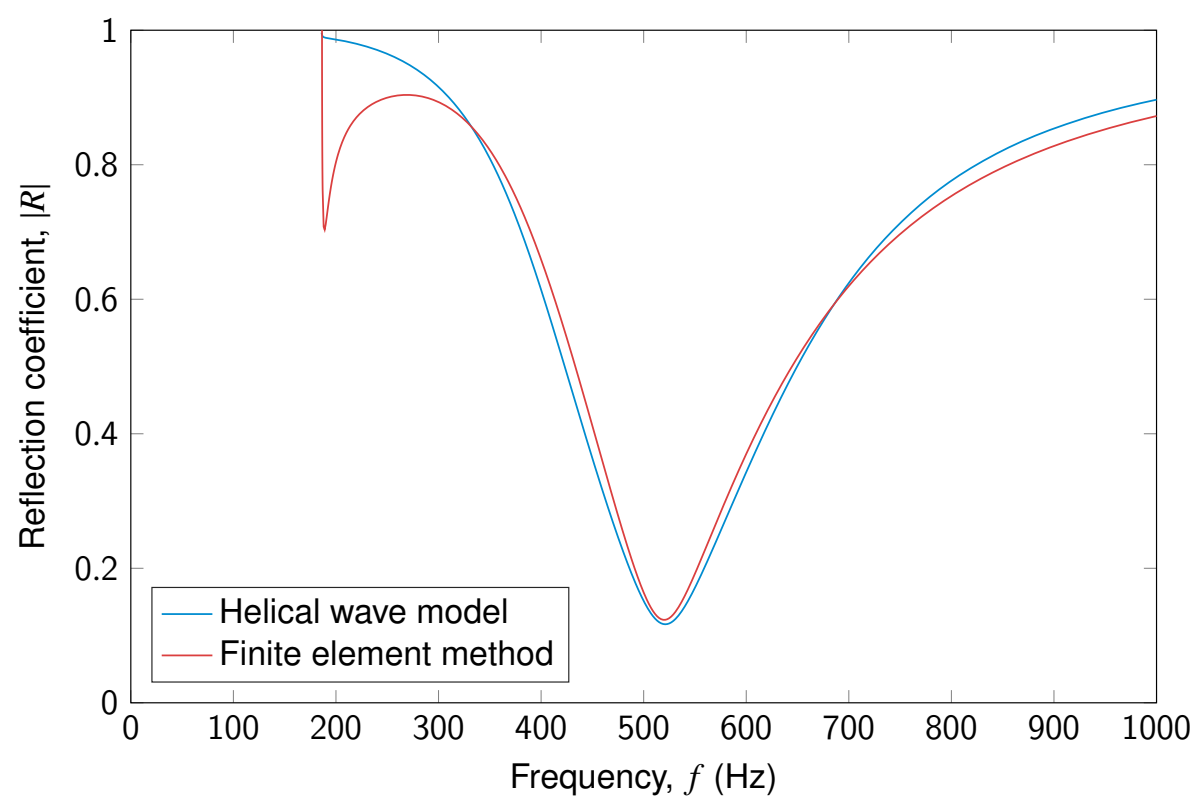

Figure 5: Comparison of the reflection coefficient of the damper from the helical wave model to a finite element method solution for an annular cavity with an incident first-order mode with no mean flow across the liner.

given by:

$$
\begin{aligned}
f_{\text {tot }}^{2} & =f_{z}^{2}+f_{\phi}^{2} \\
\Rightarrow \quad f_{\text {tot }}^{2} & =\left(\frac{c}{2 \pi} \sqrt{\left.\frac{A}{L P_{a}^{2} S}\right)^{2}+\left(\frac{c}{2 \pi} \frac{n}{r_{c}}\right)^{2}}\right. \\
\therefore \quad f_{\text {tot }} & =\frac{c}{2 \pi r_{c}} \sqrt{\frac{L n^{2} P_{a}^{2} S+A r_{c}^{2}}{L P_{a}^{2} S}}
\end{aligned}
$$

Based on a frequency of peak absorption with an incident axial wave of $495 \mathrm{~Hz}$, if the first-order circumferential mode has a cut-on frequency of $186 \mathrm{~Hz}$ the resulting total frequency is $529 \mathrm{~Hz}$ as approximately predicted by the helical wave model.

Figure 5 shows the comparison of the model against a higher-fidelity finite element method solution that was obtained using COMSOL Multiphysics. The condition presented has an incident first-order mode with no mean flow across the liner, so absorption is within the non-linear regime. The model fails to capture the initial roll-over, although the agreement is generally very good, particularly in regards to the response at resonance. The finite element method solution for this 


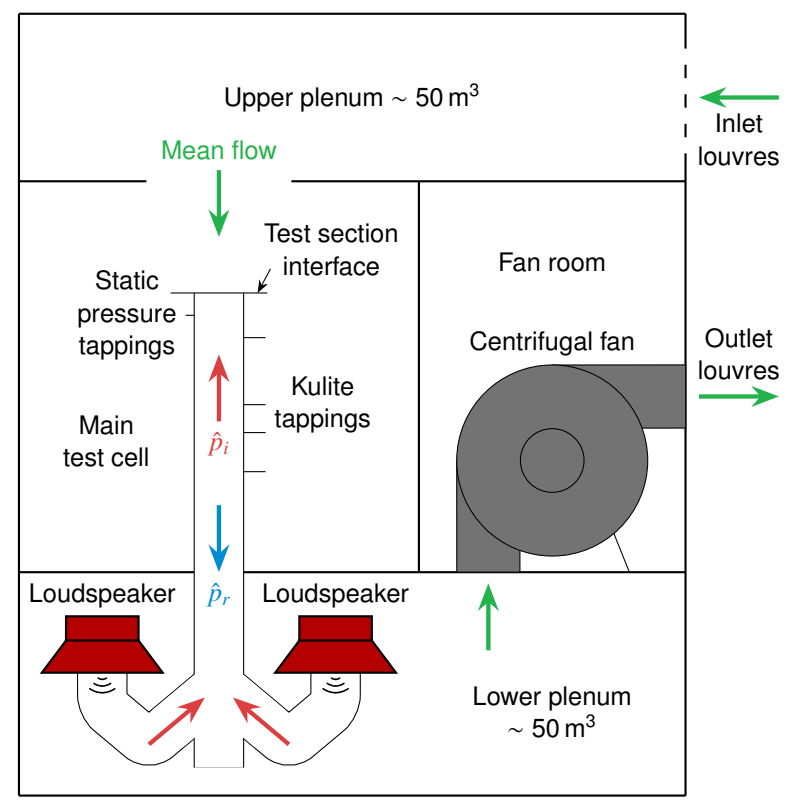

Figure 6: Simple illustration of the low intensity acoustic facility.

case took approximately 15 hours, in comparison to the time to solve the helical wave model which took less than 30 seconds. It is also dependent upon the mesh resolution of small elements, such as the finite thickness of walls, which could increase the solution time further.

\section{Experimental facility and method}

The mixed mode facility is built on an existing axial wave facility (as described in Rupp et al. [2] and Cassell [30]), which allows for small individual components to be tested when subjected to a plane wave. The isothermal test facility operates at atmospheric conditions. A simple schematic of the test facility is given in Fig. 6. Mean flow, either in the direction of propagation of the incident waves, or against them, can also be introduced. Two loudspeakers, positioned in a lower plenum, generate the acoustic waves. The annular duct for generating higher-order modes is formed from two long concentric PVC tubes as shown in Fig. 7. The ducts are mounted vertically, extending from the lower plenum into the main test cell where the test section is situated. This annulus has a mean outer diameter of $331.6 \mathrm{~mm}$ (with a peak eccentricity of $\approx 0.079$ ) and an inner diameter of $273.0 \mathrm{~mm}$; thereby, giving an average diameter of $302.3 \mathrm{~mm}$. Hence, the fundamental circumferential mode, based on the mean duct radius, occurs at approximately $360 \mathrm{~Hz}$. The radial 


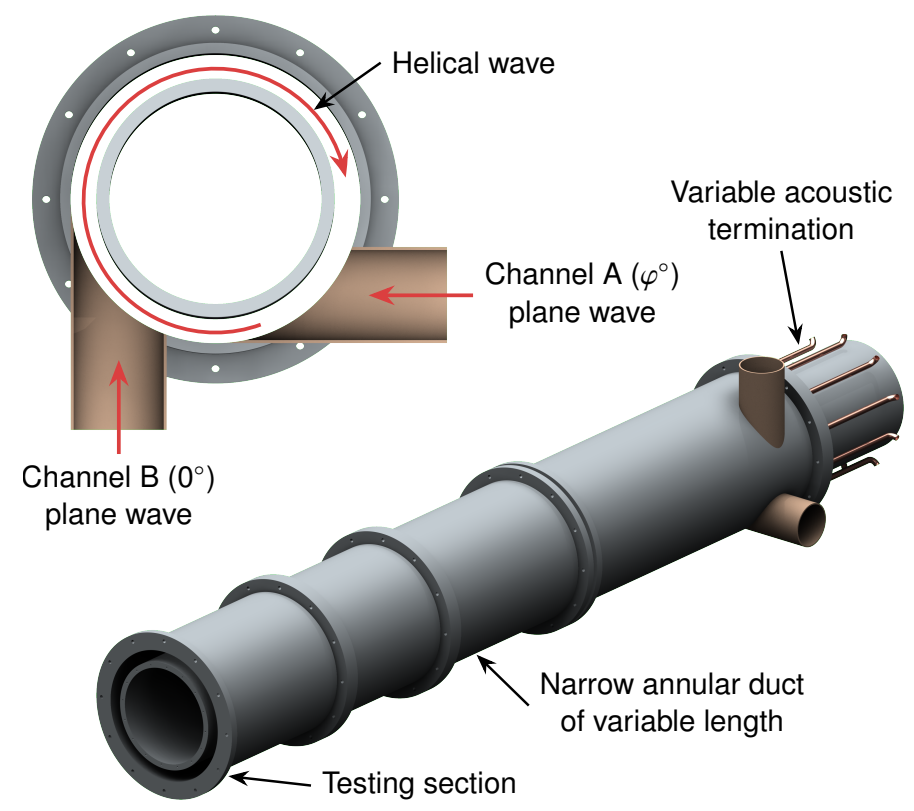

Figure 7: Low intensity mixed mode rig.

modes can be assumed to be highly cut-off and decay rapidly, with the cut-on of the radial modes estimated by [31]:

$$
f_{c}=\frac{\bar{c}}{2\left(r_{o}-r_{i}\right)} \sqrt{1-\bar{M}^{2}}
$$

Thus, with no mean flow, radial modes are predicted to cut-on at approximately $2927 \mathrm{~Hz}$ and is far greater than the frequencies considered here.

By connecting each of the loudspeakers at different circumferential locations, preference can be given to the acoustic wave structure in the duct in a method similar to that by Parrott and Watson [12] or Rademaker et al. [13]. This preference is given to the dominant circumferential mode number by the relative phase shift applied by each speaker around the circumference. A VPG608 function generator controls the phase shift between the two loudspeakers, it can generate frequencies up to $1.2 \mathrm{kHz}$; hence, up to a third-order mode can potentially be generated. Each loudspeaker has its amplitude independently set and so the experiment can be run with only a single channel operational. The excitation voltage is monitored to ensure the safe operation of the speakers within predefined limits as calculated by Biron and Simon [32]. Beyond these excitation limits the speaker response becomes non-linear; at low frequencies, this is due to limitations in the displacement of the cone and at high frequencies this is because of overheating of 
the voice coil. The aim was to generate an RMS wave amplitude of $135 \mathrm{~dB}$ for several reasons. For example, when suitably scaled, it is equivalent to the normal safe operating condition of a gas turbine combustor. In addition, it provides an acceptable signal-to-noise ratio. However, at several frequencies this was not always possible due to the limitations in excitation voltage.

Adjustments to the length of the duct can be achieved by removing or relocating sections of the duct; thus, it can be used to alter the acoustic boundary conditions. At the opposing end to the testing section the duct can be left open or have an additional plate attached. This additional plate is intended to create an acoustically closed termination. However, to allow air flow to be drawn through the rig the plate has ten axisymmetric long copper pipes fitted that provide suitably high inertia to simulate a closed end.

Eight Kulite XCS-093 series miniature dynamic pressure transducers are positioned on the main duct at a suitable distance from the test section interface. Static and dynamic calibration of the transducers was carried out. Static calibration consists of connecting the transducers to a pressure vessel alongside a calibrated reference static pressure transducer and logging the voltage across a range of pressures to calculate the sensitivity of each transducer. Dynamic calibration is performed by positioning all the Kulite transducers at the same axial height in a relatively small cross-sectional area duct. This area limitation means that when an acoustic wave is generated in the duct it propagates axially as a plane wave exciting all the transducers concurrently. Therefore, any phase shift between the pressure-time trace is due to the data acquisition process and can be corrected to less than $0.52^{\circ}$.

\subsection{Perforated liner test section}

A reconfigurable double-skinned damper has been designed that mounts axially onto the end of the annular duct. The liner, as illustrated in Fig. 8, is formed of a damping skin, an inner and outer annular wall, dividing cavity walls, and a metering skin. The skin porosity and pitch-to-diameter ratios are chosen to ensure that the apertures are suitably separated to act in isolation. The inner and outer annular walls of the damper are of the same diameters as those in the main duct at 273.0 and $331.6 \mathrm{~mm}$ respectively. Thus, this keeps the circumferential frequency constant. The main cavity has a separation between the two skins of $22.3 \mathrm{~mm}$ and is equally divided into 16 sectors, each divided by a removable $2.5^{\circ}$ wall.

To investigate the effect of sector length the dividing cavity walls are removable; allowing sectors to be merged until a full annulus is formed. Because of the finite thickness of the walls, as they are removed this causes a volume increase in 


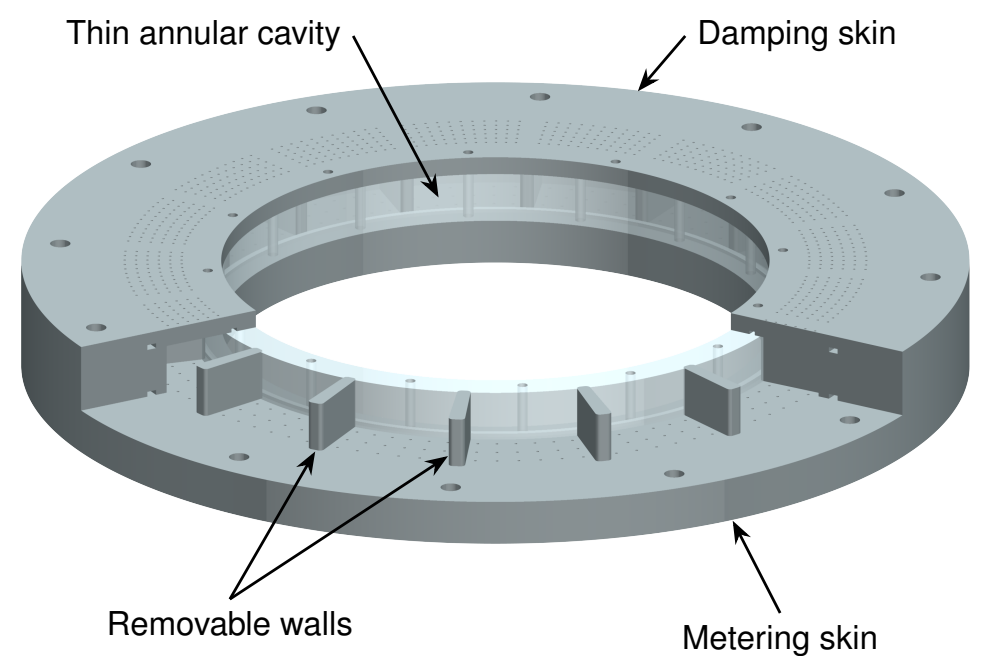

Figure 8: Perforated liner test section.

the cavity. Treating the cavity as a Helmholtz resonator, the effect of the additional volume can be quantified by the shift in resonant frequency. Based on this, the standard volume is $V=S \pi\left(r_{o}^{2}-r_{i}^{2}\right)$, and the reduced volume from having $N_{S}$ walls of $\theta$ rad each: $V=S\left(\pi-\theta N_{S}\right)\left(r_{o}^{2}-r_{i}^{2}\right)$. This results in a decrease of $46 \mathrm{~Hz}$ from having 16 walls to no walls. To aid location and minimise cross-sector leakage, the walls fit into shallow grooves in the inner and outer walls. The extra volume, added to the total because of the groove, is negligible compared to that of the wall thickness (a further $6 \mathrm{~Hz}$ decrease). The predicted responses of the liner with an incident axial or helical wave are shown in Fig. 9 using the resonance parameter to normalise the frequency. A value of one in the resonance parameter corresponds to the theoretical resonant frequency, which for the double perforated skin system is equated to a Helmholtz resonator with two throats as described by Keller and Zauner [33]:

$$
f=\frac{c}{2 \pi} \sqrt{\frac{1}{V}\left(\frac{\pi r_{1}^{2}}{L_{1}}+\frac{\pi r_{2}^{2}}{L_{2}}\right)}
$$

Thus, the resonance parameter is defined as [3]:

$$
Q_{r}=\frac{k_{0}^{2}}{\frac{\pi r_{2}^{2}}{L_{2} P_{a_{1}^{2}}^{2} S}+\frac{\pi r_{2}^{2}}{L_{2} P_{a_{2}^{2}}^{2} S}}
$$

The two responses with an axial wave both resonate with a similar response as 


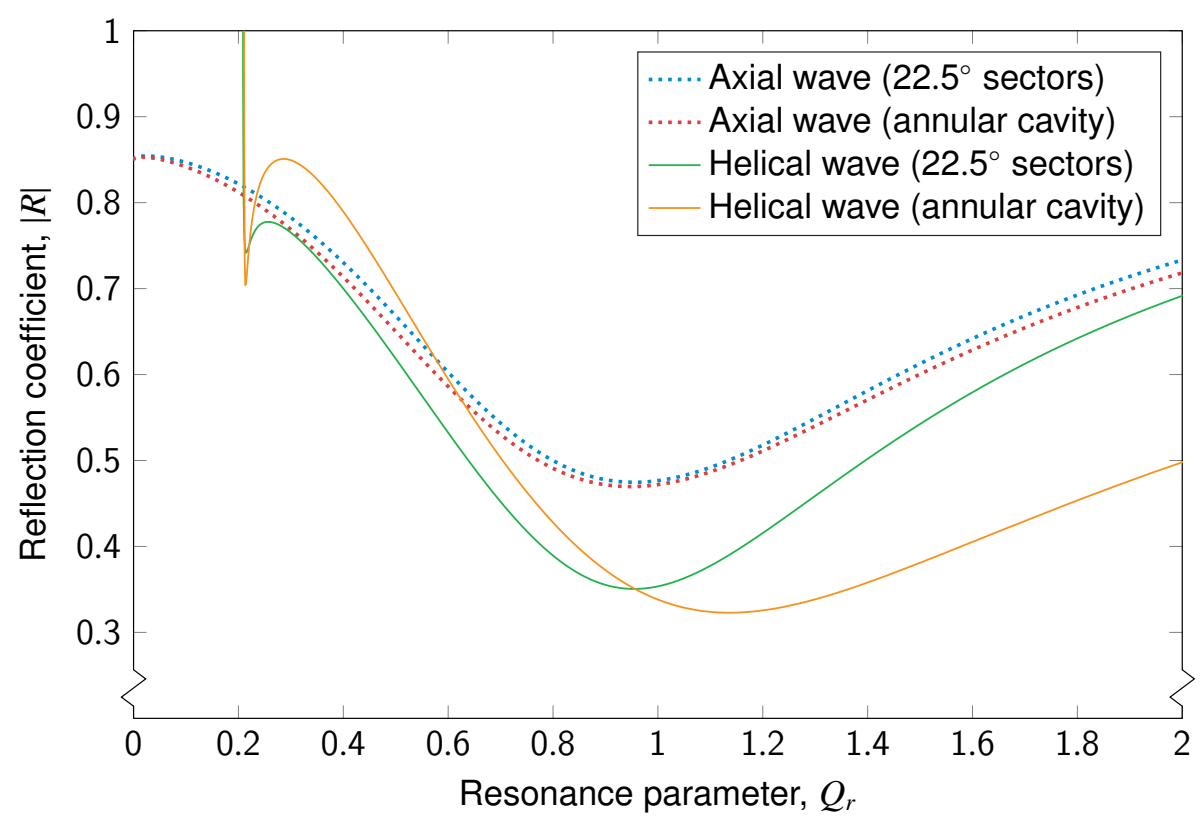

Figure 9: Predicted reflection coefficient (defined per Eq. 26) of the $22.5^{\circ}$ sector and annular configurations against resonance parameter for the experimental rig using the helical wave model.

expected, although with a slight difference in the reflection coefficient due to the cavity volume difference. As modelled previously, with an incident helical wave the $22.5^{\circ}$ sector configuration resonates at a similar frequency to the incident axial wave, although the large annular cavity generates a internal circumferential mode shape shifting the resonant frequency higher than that predicted by Eq. 29.

A frequency increase is observed between the predicted response of the annular cavity relative to the $22.5^{\circ}$ sector with an incident helical wave that is not present with an incident axial wave because of the formation of mode shapes within the cavity. Equation 29 calculates a resonant frequency that is different to that predicted by the model, due to the mean flow that is applied across the damper.

\subsection{Higher-order multi-microphone method}

For an axial wave, it is assumed the total fluctuation is based on the superposition of up to two waves of the same frequency; an incident and a reflected wave. However, for a helical wave of a single mode, the fluctuation at a single frequency may be the linear superposition of up to four individual waves as illustrated in Fig. 10, with two reflected and two incident waves travelling either clockwise or anti-clockwise directions. The following method assumes that the 


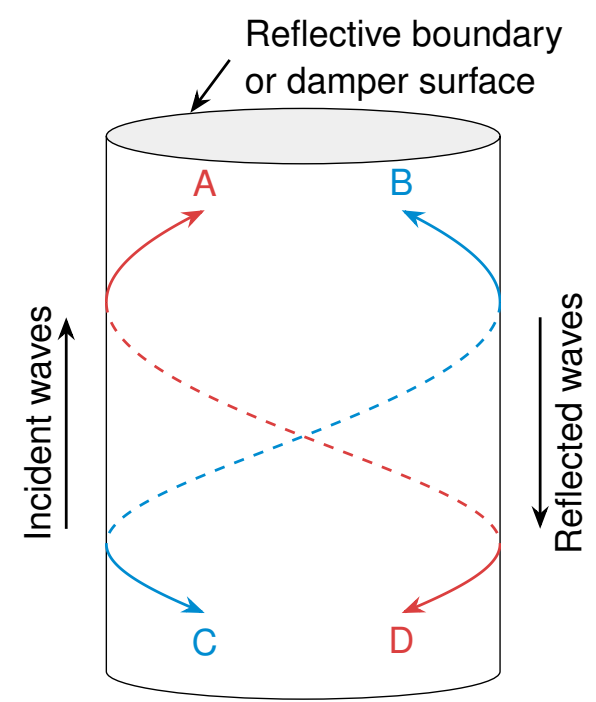

Figure 10: Four possible waves in a single circumferential mode.

interaction of different waves and modes within the entire field can be described using the principle of linear superposition. It is acknowledged however, in the very near field of the damper face, it may invalidate this principle as the field may become acoustically coupled. Assuming the waves can always be described with a linearised method allows for an initial comparison of these conditions; however, the error associated with the decomposition process may increase.

To calculate the four potential amplitudes for a single mode the higher-order multi-microphone method software solves $\mathbf{A x}=\mathbf{b}$ for a system using $m$ microphones where the terms are:

$$
\begin{aligned}
& \mathbf{A}=\left[\begin{array}{cccc}
\mathrm{e}^{\mathrm{i}\left(k_{z}^{+} z_{1}+\phi_{1} n+\varphi_{n}\right)} & \mathrm{e}^{\mathrm{i}\left(k_{z}^{+} z_{1}-\phi_{1} n+\varphi_{n}\right)} & \mathrm{e}^{\mathrm{i}\left(k_{z}^{-} z_{1}+\phi_{1} n+\varphi_{n}\right)} & \mathrm{e}^{\mathrm{i}\left(k_{z}^{-} z_{1}-\phi_{1} n+\varphi_{n}\right)} \\
\mathrm{e}^{\mathrm{i}\left(k_{z}^{+} z_{2}+\phi_{2} n+\varphi_{n}\right)} & \mathrm{e}^{\mathrm{i}\left(k_{z}^{+} z_{2}-\phi_{2} n+\varphi_{n}\right)} & \mathrm{e}^{\mathrm{i}\left(k_{z}^{-} z_{2}+\phi_{2} n+\varphi_{n}\right)} & \mathrm{e}^{\mathrm{i}\left(k_{z}^{-} z_{2}-\phi_{2} n+\varphi_{n}\right)} \\
\vdots & \vdots & \vdots & \vdots \\
\mathrm{e}^{\mathrm{i}\left(k_{z}^{+} z_{\mathrm{m}}+\phi_{\mathrm{m}} n+\varphi_{n}\right)} & \mathrm{e}^{\mathrm{i}\left(k_{z}^{+} z_{\mathrm{m}}-\phi_{\mathrm{m}} n\right)+\varphi_{n}} & \mathrm{e}^{\mathrm{i}\left(k_{z}^{-} z_{\mathrm{m}}+\phi_{\mathrm{m}} n+\varphi_{n}\right)} & \mathrm{e}^{\mathrm{i}\left(k_{z}^{-} z_{\mathrm{m}}-\phi_{\mathrm{m}} n+\varphi_{n}\right)}
\end{array}\right], \\
& \mathbf{x}=\left[\begin{array}{c}
\hat{p}_{A} \\
\hat{p}_{B} \\
\hat{p}_{C} \\
\hat{p}_{D}
\end{array}\right], \quad \mathbf{b}=\left[\begin{array}{c}
p_{1}^{\prime} \\
p_{2}^{\prime} \\
\vdots \\
p_{\mathrm{m}}^{\prime}
\end{array}\right]
\end{aligned}
$$

where the column vector $\mathbf{x}$ is the four wave amplitudes to be calculated, and $\mathbf{b}$ is the pressure measured by each microphone. Matrix A is formed from the known microphone positions ( $z$ and $\phi$ ), and the axial wave number that varies based on 
the mean flow direction relative to the incident wave according to [31]:

$$
k_{z}^{ \pm}=\frac{\omega \bar{M} \pm \sqrt{\omega^{2}-\frac{\bar{c}^{2} n^{2}}{r_{\mathrm{avg}}^{2}}\left(1-\bar{M}^{2}\right)}}{\bar{c}\left(1-\bar{M}^{2}\right)}
$$

The software attempts to solve for the least squares solution of $\mathbf{x}$ that minimises $\|\mathbf{b}-\mathbf{A x}\|^{2}$ for each possible circumferential mode value (e.g. $\left.n=\{0,1,2, \ldots\}\right)$ or combination of simultaneous modes. The combination of wave amplitudes, mode number, and phase that result in the lowest residuals are hence taken as being the best solution.

Synthetic data was generated across a range of operating conditions and mode structures to assess the errors produced by the decomposition software. During this process, the dependency on the number of microphones was studied to determine the minimum number required to accurately decompose the field [30]. Additionally, the placement of the microphones was assessed, which was initially based on the routine described by Pickett et al. [34, 35]. With a circumferentially travelling acoustic field, which will not be presented here, the errors were minimised using this optimisation routine. Although for a dominantly circumferentially standing acoustic field, which is the focus of this paper, these layouts provided negligible benefit and instead an L-shaped layout of microphones was utilised for convenience.

A flat plate mounted across the duct termination to reflect the acoustic energy was used to establish the potential error from the measurement and decomposition process, as the closed end should provide a reflection coefficient of one. The results for both a circumferentially standing and spinning field are presented in Fig. 11 show that the experimental uncertainty for the total response over the frequency range of interest, is on average below $10 \%$. The error increases with frequency as it becomes increasingly difficult to resolve the acoustic field with the limited number of microphones used. Peaks in the decomposed mode errors occur close to the cut-on of the circumferential modes, and could be due to the transfer of energy between modes at these conditions or error associated with the decomposition method. This causes the reflection coefficient values higher than one in the decomposed modes.

\section{Acoustic absorption measurements}

An example data set is presented here with the speakers in-phase with each other producing a nominally circumferentially standing acoustic field where the 


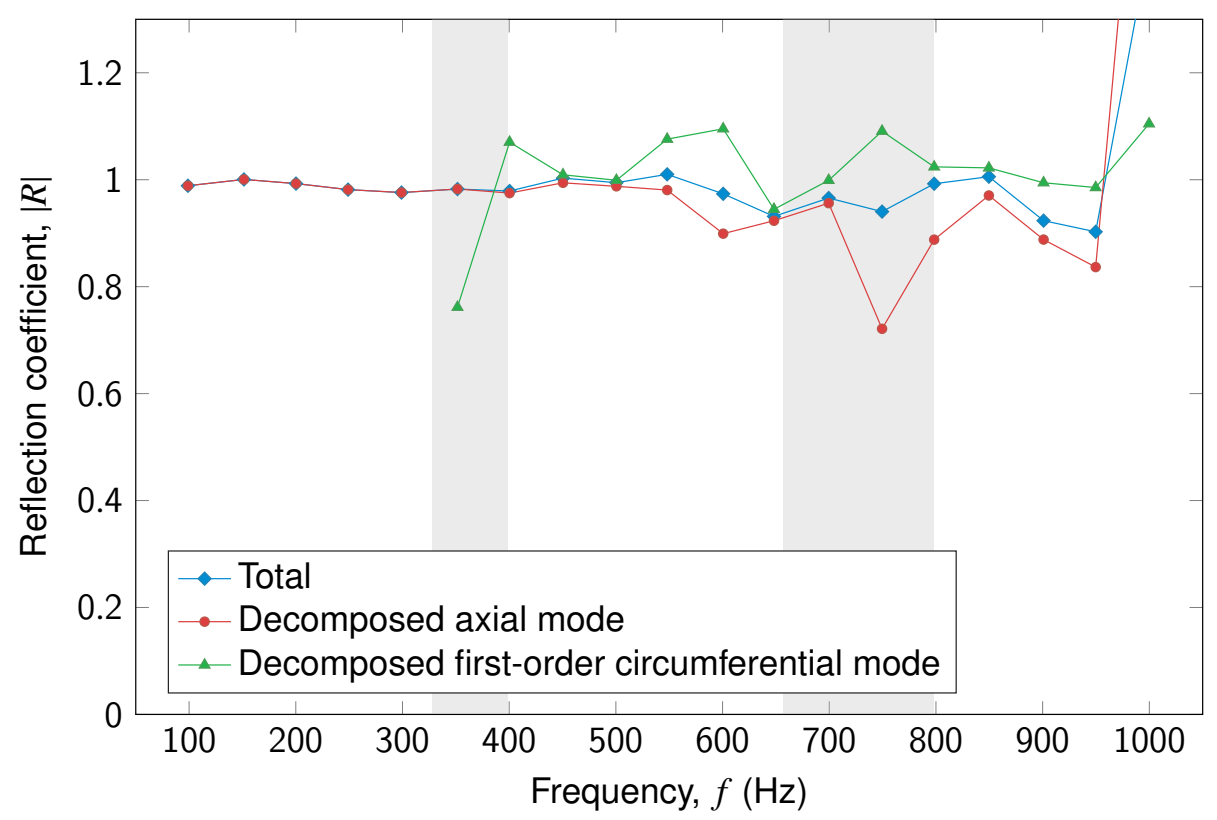

(a)

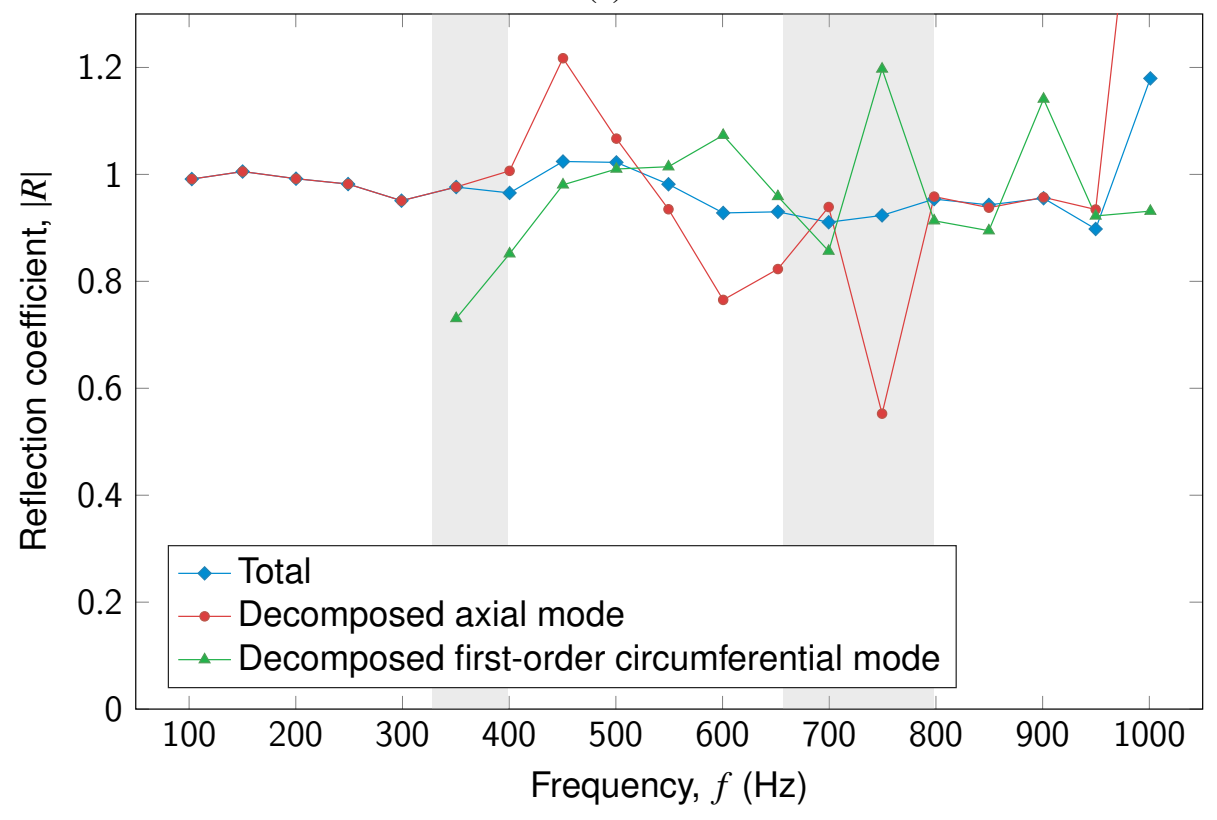

(b)

Figure 11: Reflection coefficients of the total and decomposed acoustic modes with frequency with a closed duct termination. (a) circumferentially standing and (b) circumferentially travelling acoustic field. The grey shaded regions indicate the possible frequency ranges of first- and second-order circumferential mode cut-on. 
axial mode dominates the acoustic field. Analysis is performed with respect to variations in liner pressure drop and the number of cavity divisions within the damper. The same analysis has also been applied to a larger data set across a range of axial and circumferential component combinations although these are not presented here for clarity. As with a circumferentially spinning mode the field is more susceptible to errors during decomposition that can arise from acoustic modal coupling.

The magnitude and phase of the measured reflection coefficient are presented in Figs. 12 and 13 respectively for an annular cavity with a total pressure drop $\left(\Delta \bar{p} / p_{0}\right)$ between 0 and $4 \%$. Figure 12(a) presents the total combined reflection coefficient of the cut-on acoustic modes, similarly Fig. 13(a) shows the phase for these modes. This includes only the modes that have cut-on across the radial variation of the duct and thus do not have an attenuation rate which causes the mode to decay noticeably as it propagates to the measurement locations. This total response is a function of the magnitudes of the different modes present (in the current configuration the axial mode generally dominates this response with on average $59.9 \%$ of the total incident pressure amplitude) and has less inherent error than the decomposed modes. The lightly shaded regions illustrate the possible range in frequencies of cut-on for the first- and second-order modes. This cut-on frequency range is a result of the annular geometry, with the outer wall having the first-order mode cut-on at $328 \mathrm{~Hz}$ and the inner wall cutting-on at $399 \mathrm{~Hz}$. Because of the uncertainty and variation in cut-on, it therefore means that the error can be higher in this region. Through the cut-on region the instability of the mode causes a local minimum in the associated modal reflection coefficient as the mode can still decay. At a frequency $50-100 \mathrm{~Hz}$ higher than the inner wall cut-on (450-500 Hz for the first-order mode) the reflection coefficient tends to a value of one indicating the mode has cut-on throughout the acoustic field.

There is a clear disparity in the magnitude of the reflection coefficient shown in Fig. 12 between a total pressure drop of 0 and $1 \%$, due to the transition from non-linear to linear absorption. With further increases in pressure drop, that are also in the linear absorption regime, a slight decrease in absorption is observed for both modes; although, this is most evident in the axial mode. Variations in incident pressure amplitude may cause non-linear absorption at some frequencies. An example of this non-linearity is observed for all pressure drop conditions at 575 and $600 \mathrm{~Hz}$. The relative phases and amplitudes of the circumferential and axial modes cause an interference pattern around the circumference, which can have sufficient pressure amplitude to cause non-linear absorption over part of the skin. However, while this region of non-linear absorption is noticeable 


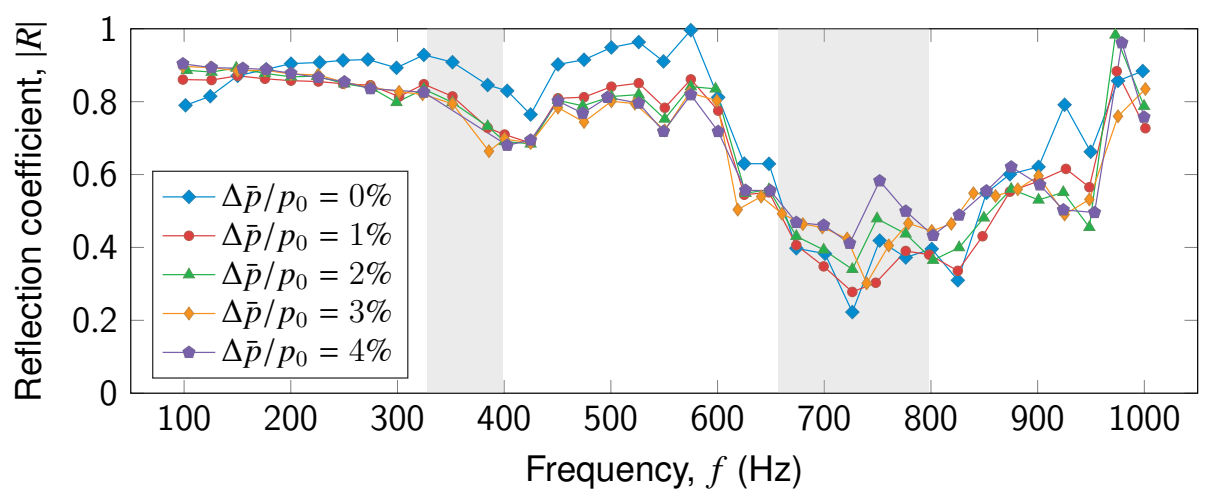

(a)

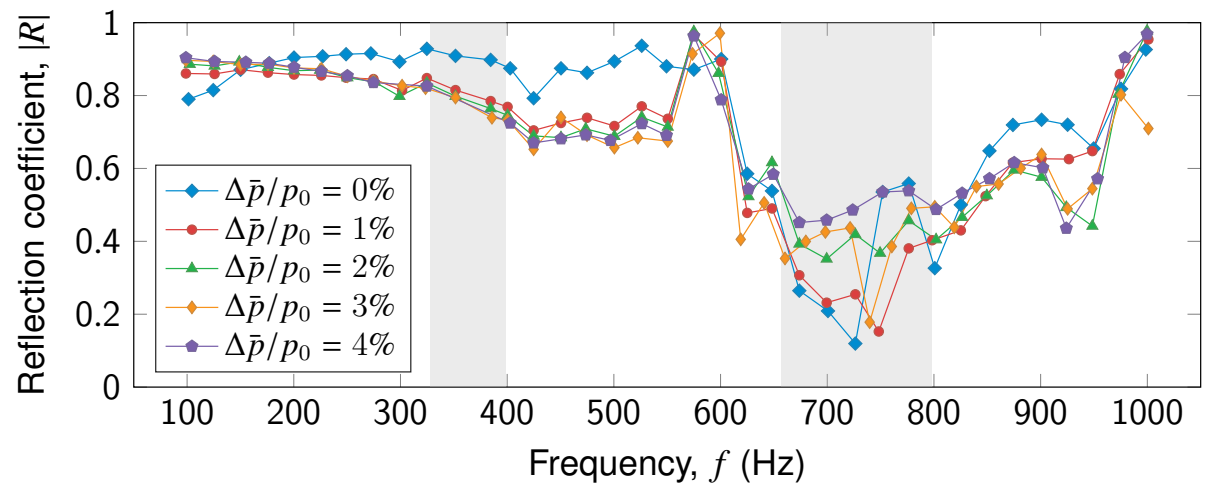

(b)

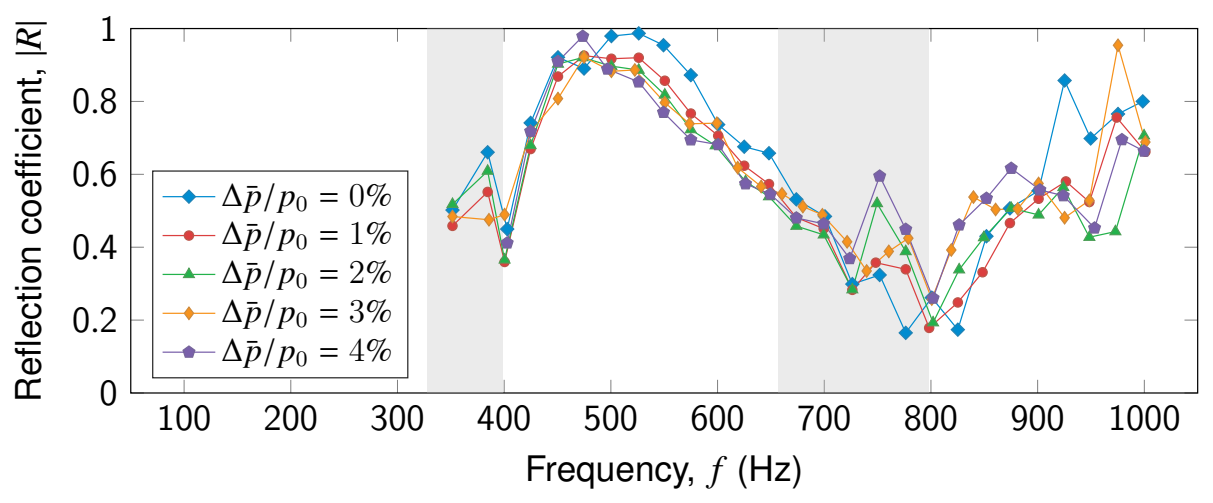

(c)

Figure 12: Reflection coefficient with frequency for the annular cavity with a liner pressure drop of $0-4 \%$ with a dominant incident axial mode $\left(\hat{p}_{i}=135 \mathrm{~dB}\right)$. (a) the total (cut-on modes) field response, (b) the axial mode response, and (c) the first-order circumferential mode response. The grey shaded regions indicate the possible frequency ranges of first- and second-order circumferential mode cut-on. 


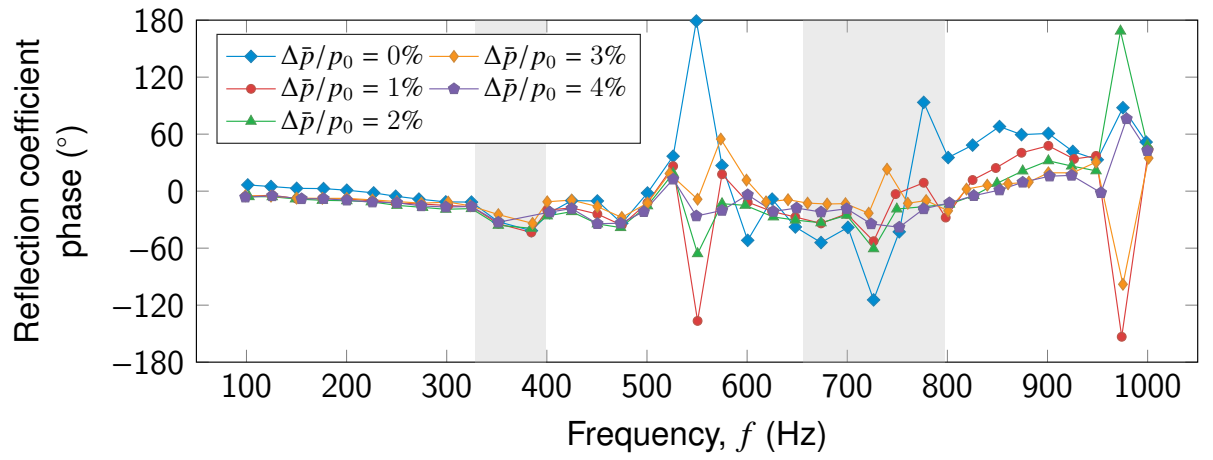

(a)

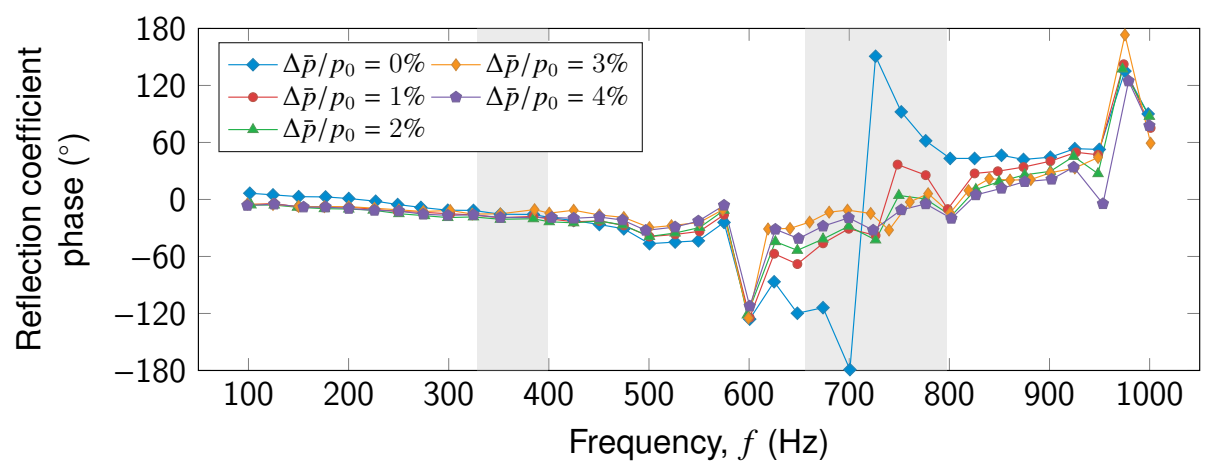

(b)

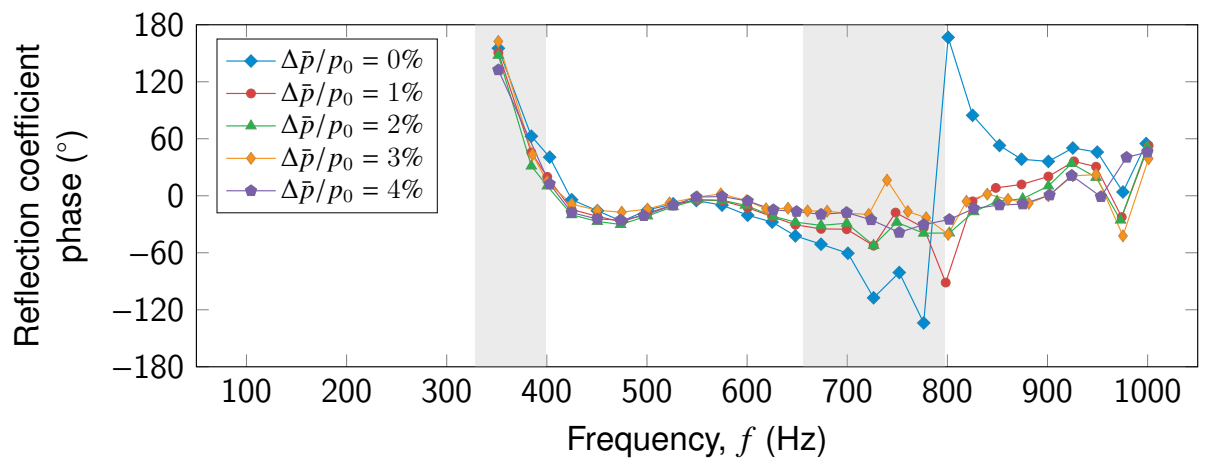

(c)

Figure 13: Reflection coefficient phase with frequency for the annular cavity with a liner pressure drop of $0-4 \%$ with a dominant incident axial mode $\left(\hat{p}_{i}=135 \mathrm{~dB}\right)$. (a) the total (cut-on modes) field response, (b) the axial mode response, and (c) the first-order circumferential mode response. The grey shaded regions indicate the possible frequency ranges of first- and second-order circumferential mode cut-on. 
in the axial mode response (Fig. 12(b)), no distinct variation is observed in the circumferential mode (Fig. 12(c)). This is due to the circumferential variation in acoustic pressure for the circumferential mode, such that while one portion of the skin may be non-linear, others are within the linear absorption regime, and thus when averaged is not noticeable.

By decomposing the acoustic field, the amplitudes of the axial and each circumferential mode can be calculated. Thus, the reflection coefficients calculated for the axial and first-order circumferential modes are shown in Figs. 12(b) and 12(c). The decomposition software calculates that a second-order circumferential mode has cut-on within the duct at frequencies above $700 \mathrm{~Hz}$, although with a relatively low magnitude over the frequency range of interest (and is thus not plotted). The frequency of minimum reflection coefficient is observed to increase from the axial mode at approximately $720 \mathrm{~Hz}$ to the first-order circumferential mode at $800 \mathrm{~Hz}$. This is a result of the added circumferential component as, using Eq. 27, it is expected a frequency increase will occur from $720 \mathrm{~Hz}$ to $805 \mathrm{~Hz}$ based on the mean cut-on frequency. This increase is supported by the phase of the reflection coefficient (shown in Figs. 13(b) and 13(c)) and is particularly noticeable in the non-linear case where at resonance a change in sign that occurs. Plotting the first-order circumferential mode with axial frequency against the axial mode results in the comparison given in Fig. 14(b) and demonstrates that the data largely collapses as expected (except at $600 \mathrm{~Hz}$ when non-linear effects are dominant in the axial mode). The axial frequency error bars are based on the inner and outer duct diameters. The data are also cross-plotted with total frequency in Fig. 14(a). For the circumferential mode the associated axial frequency component is derived from the total frequency as a pure spinning wave, based on the mean duct radius. While only the data for a pressure drop of $1 \%$ are shown for clarity, all the cases share similar trends. The data generally collapse better when plotted against the axial frequency component; although, with a slight disparity due to the variation in cut-on frequency but is within the limits of experimental error.

\subsection{Cavity segmentation}

The removable walls in the cavity, illustrated in Fig. 8, allow the configuration to be varied from 16 individual sectors to a fully annular cavity. The walls are removed to preserve symmetry and keep the sector lengths uniform. Six configurations are tested; 16 walls to form 16 sectors of approximately $22.5^{\circ}$ each, 8 walls resulting in $45^{\circ}$ per sector, 4 walls for four $90^{\circ}$ sectors, 2 walls with two $180^{\circ}$ sectors, a single wall producing a $360^{\circ}$ sector, and the fully annular configuration. A peak absorption frequency increase, of around $50 \mathrm{~Hz}$, is expected due to the 


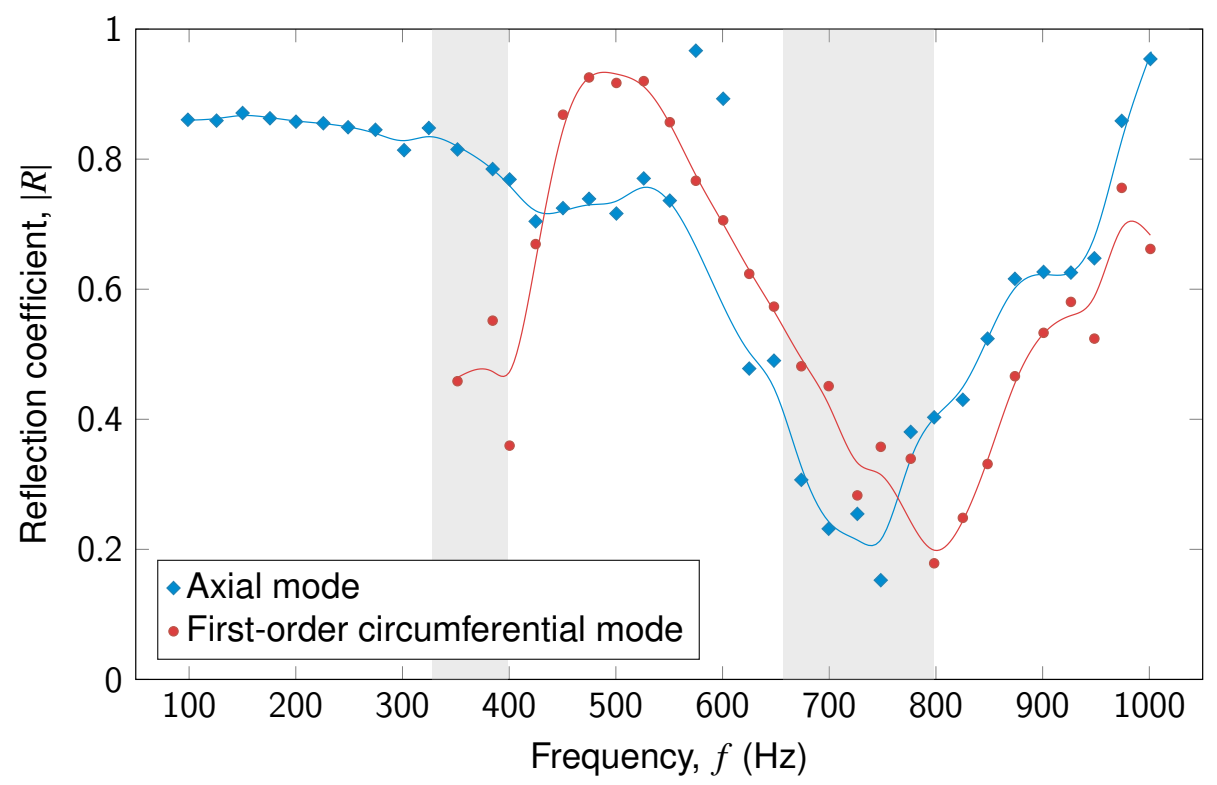

(a)

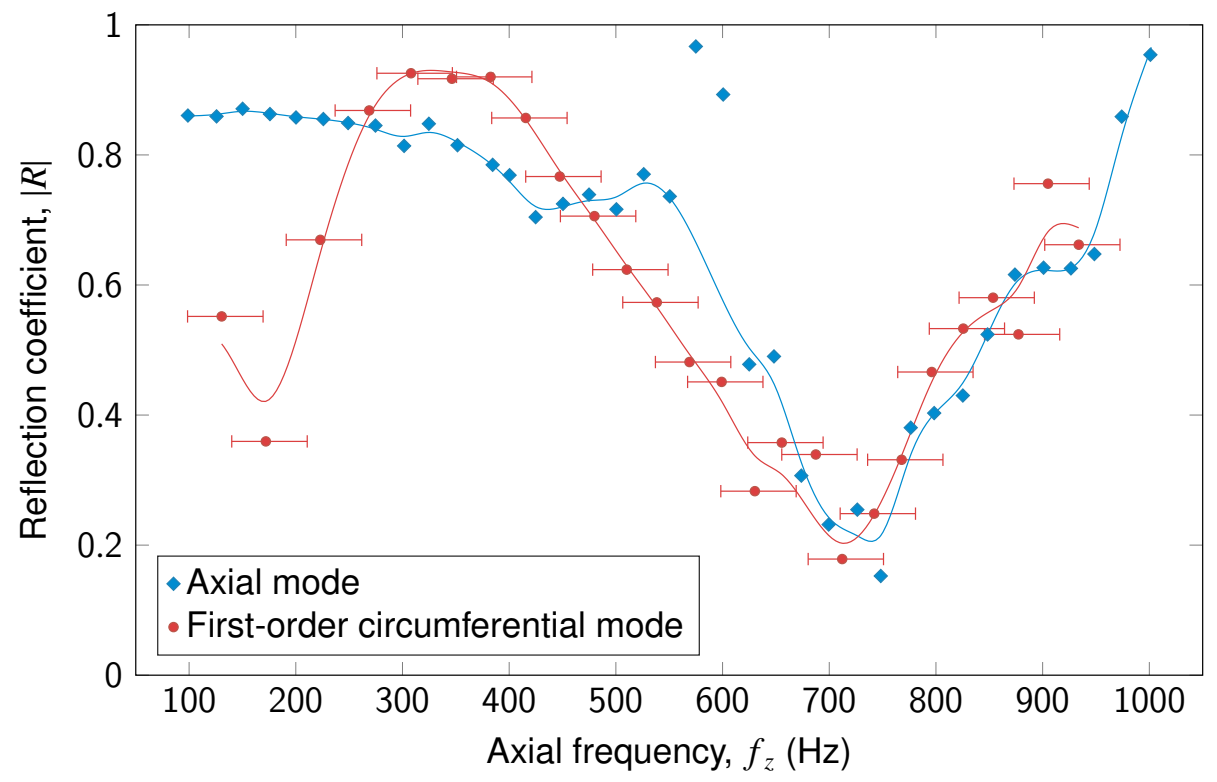

(b)

Figure 14: Reflection coefficient for the axial and first-order circumferential decomposed modes for the annular cavity with a total pressure drop of $1 \%$ with: (a) the total frequency and (b) the axial frequency component. The axial frequency error bars are based on the inner and outer duct diameters. 
finite thickness of the walls between the $22.5^{\circ}$ and annular configurations. The experimental results presented have been conducted with a $3 \%$ total liner pressure drop, and thus the damper is expected to generally operate in the linear absorption regime.

Figure 15 presents the magnitude of the reflection coefficients obtained for the different configurations. The reflection coefficients presented represent: (a) the total response from the cut-on modes, (b) the decomposed response of the axial mode, and (c) the decomposed response of the first-order circumferential mode. Figure 16 shows the phase of the reflection coefficients, for the total and decomposed axial and helical modes. It can be initially observed from the reflection coefficient responses that the axial mode fills each cavity uniformly and is independent of how many segments are present. Thus, cavity segmentation has no effect on the acoustic response, aside from as mentioned, the small frequency variation due to the volume change associated with the presence/removal of the segment walls. The first-order circumferential mode response shows there is a noticeable increase in the frequency at which peak absorption occurs as the number of cavity segmentations is reduced. This frequency increase causes an appreciable disparity in reflection coefficient between 500 and $700 \mathrm{~Hz}$. The 22.5 , 45 , and $90^{\circ}$ configurations reach a peak noted at approximately $725 \mathrm{~Hz}$, whereas the $180,360^{\circ}$, and annular configurations are suggested to have a peak at a higher frequency. From the previous study of pressure drop for the annular cavity, illustrated in Figs. 12, 13, and 14(a), this frequency of resonance should be at approximately $800 \mathrm{~Hz}$. This increase for the circumferentially long cavities is related to the circumferential frequency, which results in a mode shape being generated within the cavity circumferentially. Non-linear absorption occurs within the axial mode at 575 and $600 \mathrm{~Hz}$ for all the cavity configurations.

When the first-order mode initially cuts-on, a low reflection coefficient is initially observed. This is due to the variation in the cut-on frequency through the annular height potentially producing a cut-off mode that has a high attenuation rate and decays rapidly as it propagates. The circumferentially compact configurations $\left(22.5,45\right.$, and $\left.90^{\circ}\right)$ have greater loss measured between 500 and $700 \mathrm{~Hz}$. This is attributable to the resonance of the cavity being dependent upon the axial frequency component. For the circumferentially compact systems, the cavity axial frequency is approximately the total frequency $\left(f_{\text {tot }} \approx f_{z_{c}}\right)$. Alternatively, when circumferential cavity mode shapes are generated the axial frequency component of the cavity is now based upon the circumferential frequency component $\left(f_{\text {tot }}^{2}\right.$ $f_{\phi_{c}}^{2} \approx f_{z_{c}}^{2}$ ); hence, cavity resonance occurs at a higher frequency. As a result, the loss measured for the circumferentially long configurations is lower over the 500- 


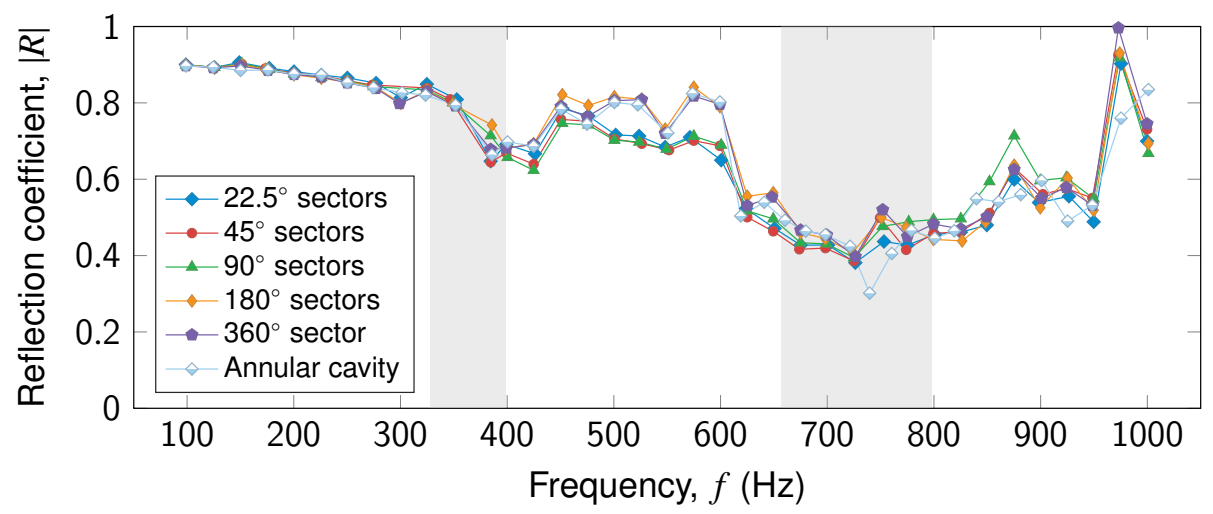

(a)

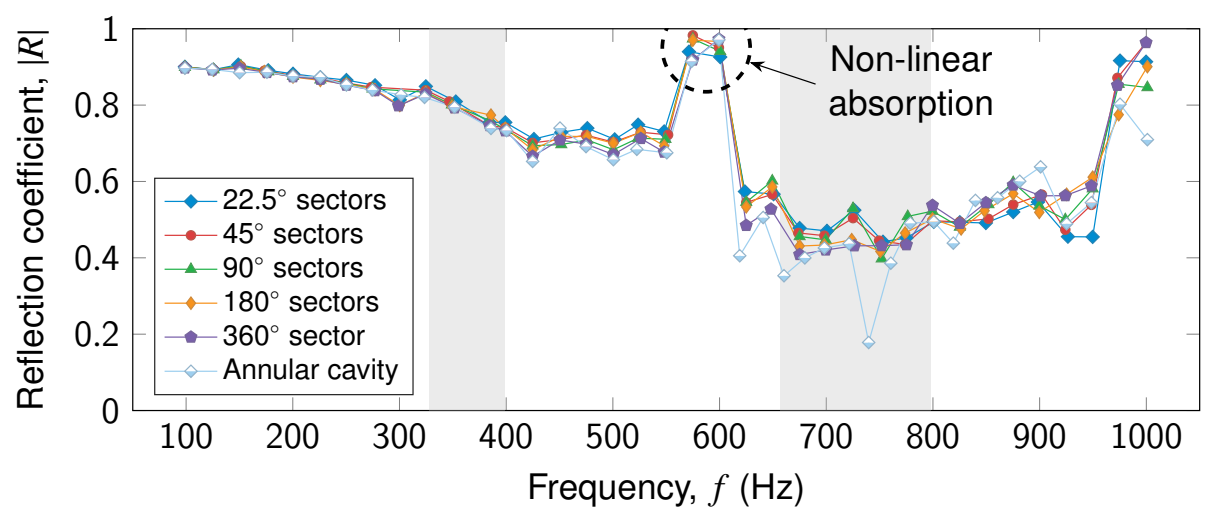

(b)

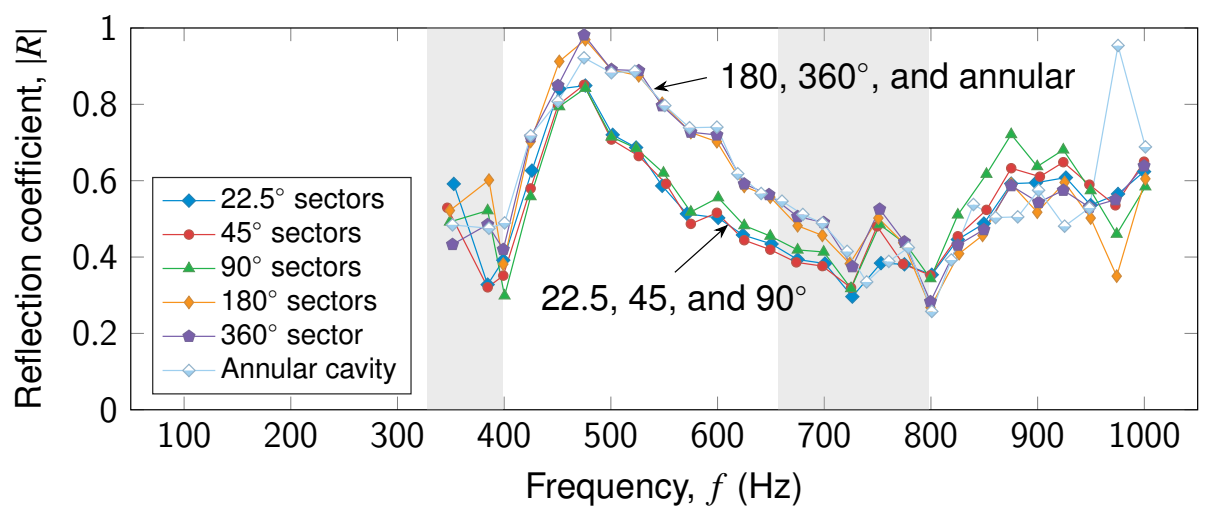

(c)

Figure 15: Effect of cavity segmentation on the reflection coefficient with frequency with a dominant axial mode $\left(\Delta \bar{p} / p_{0}=3 \%\right)$. (a) the total (cut-on modes) field response, (b) the axial mode response, and (c) the first-order circumferential mode response. The grey shaded regions indicate the possible frequency ranges of first- and second-order circumferential mode cut-on. 


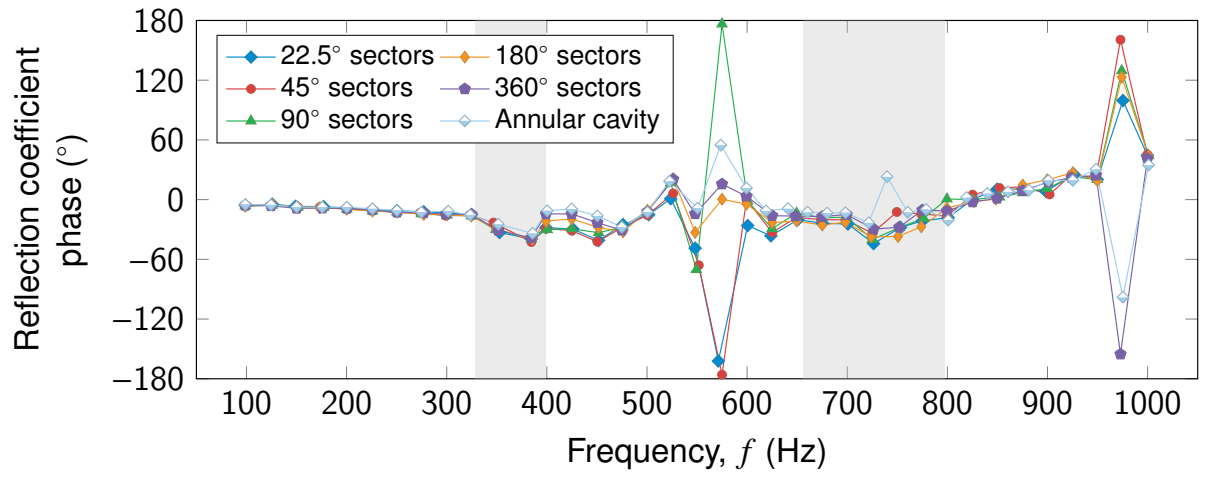

(a)

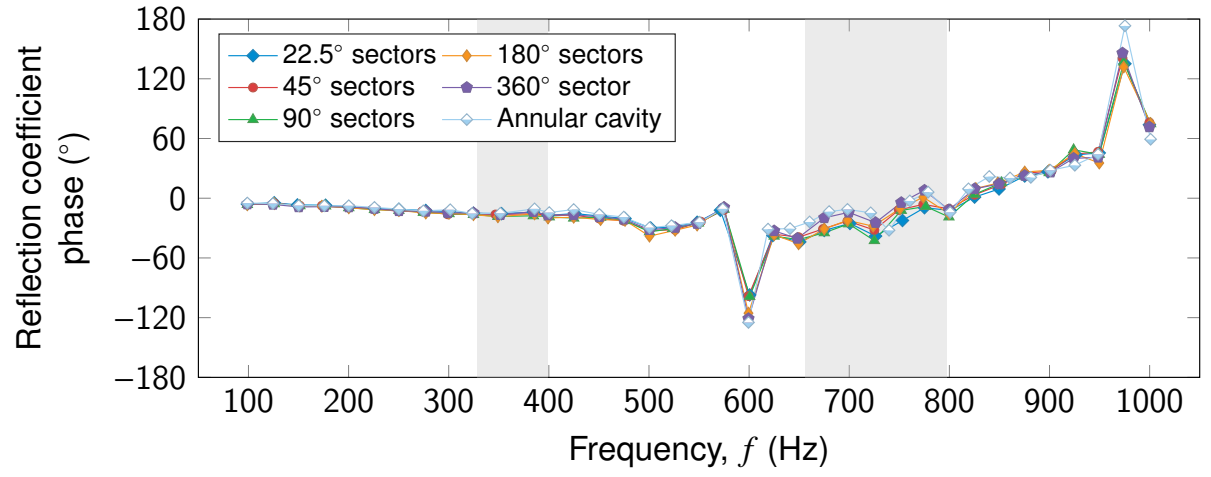

(b)

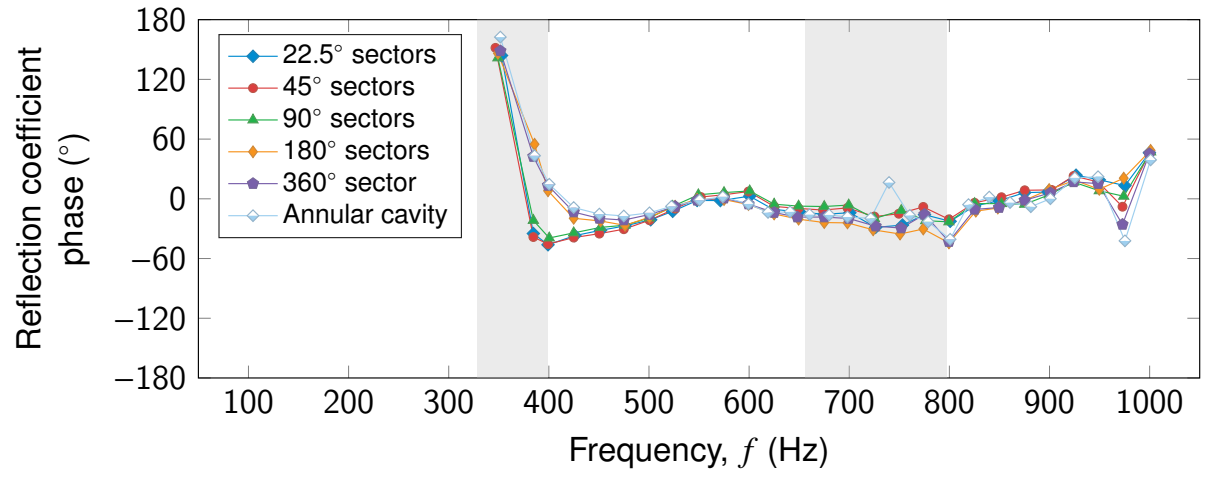

(c)

Figure 16: Effect of cavity segmentation on the reflection coefficient phase with frequency with a dominant axial mode $\left(\Delta \bar{p} / p_{0}=3 \%\right)$. (a) the total (cut-on modes) field response, (b) the axial mode response, and (c) the first-order circumferential mode response. The grey shaded regions indicate the possible frequency ranges of first- and second-order circumferential mode cut-on. 
$700 \mathrm{~Hz}$ range, as it is comparatively further from resonance. As the total frequency increases away from circumferential cut-on, this disparity in loss reduces as the axial frequency for that mode tends to the total for all configurations.

As plotted previously (Fig. 14) the axial and circumferential modes can be shown to broadly collapse when plotted using the axial frequency component of the helical cavity wave instead of the total if the cavity is not compact (180, $360^{\circ}$, and annular). Presenting the reflection coefficient of the $22.5^{\circ}$ and annular configurations in this way yields Figs. 17 and 18 respectively. The reflection coefficient for each mode is the ratio of the reflected and incident pressure amplitudes associated with the mode. The annular configuration has a cavity length that is long relative to the circumferential wavelength and produces a total frequency shift relative to the axial mode response. However, the $22.5^{\circ}$ configuration does not generate this total frequency shift. This is because the pressure distribution across the liner for the $22.5^{\circ}$ configuration is sufficiently small such that it can be initially considered as being equivalent to an incident axial wave. Hence, this does not result in a collapse of the data when plotted against axial frequency component and, instead, generally better agreement is achieved with total frequency for this configuration. The response of the annular (Fig. 18(b)) collapses well against the axial frequency component, as would also the 180 and $360^{\circ}$ configurations; although, disparity is noted due to the uncertainty in the circumferential mode cut-on frequency. The limits of the error bars are determined from the cut-on of the inner and outer annulus walls. As Fig. 19 shows the first-order circumferential mode data collapses with good agreement when each sector configuration is plotted against the relevant frequency component, although with some disparity because of the difference in cavity volume.

\section{Comparison of experimental results to analytical model}

Figure 20 presents a comparison between the axial wave model and the decomposed axial mode results that were presented in Fig. 15(b). Reasonable agreement is obtained for all the configurations against the axial wave model using theoretical length correction and discharge coefficients. The axial wave model predicts a shift in resonant frequency related to the increase in cavity volume by the reducing number of dividing walls, although practically this shift is difficult to observe.

A comparison is given in Fig. 21 for the decomposed first-order circumferential mode results, presented originally in Fig. 15(c) with a relative speaker phase of zero, against the axial and helical wave models. The helical wave model is generally in very good agreement for the acoustically compact $\left(22.5,45\right.$, and $\left.90^{\circ}\right)$ 


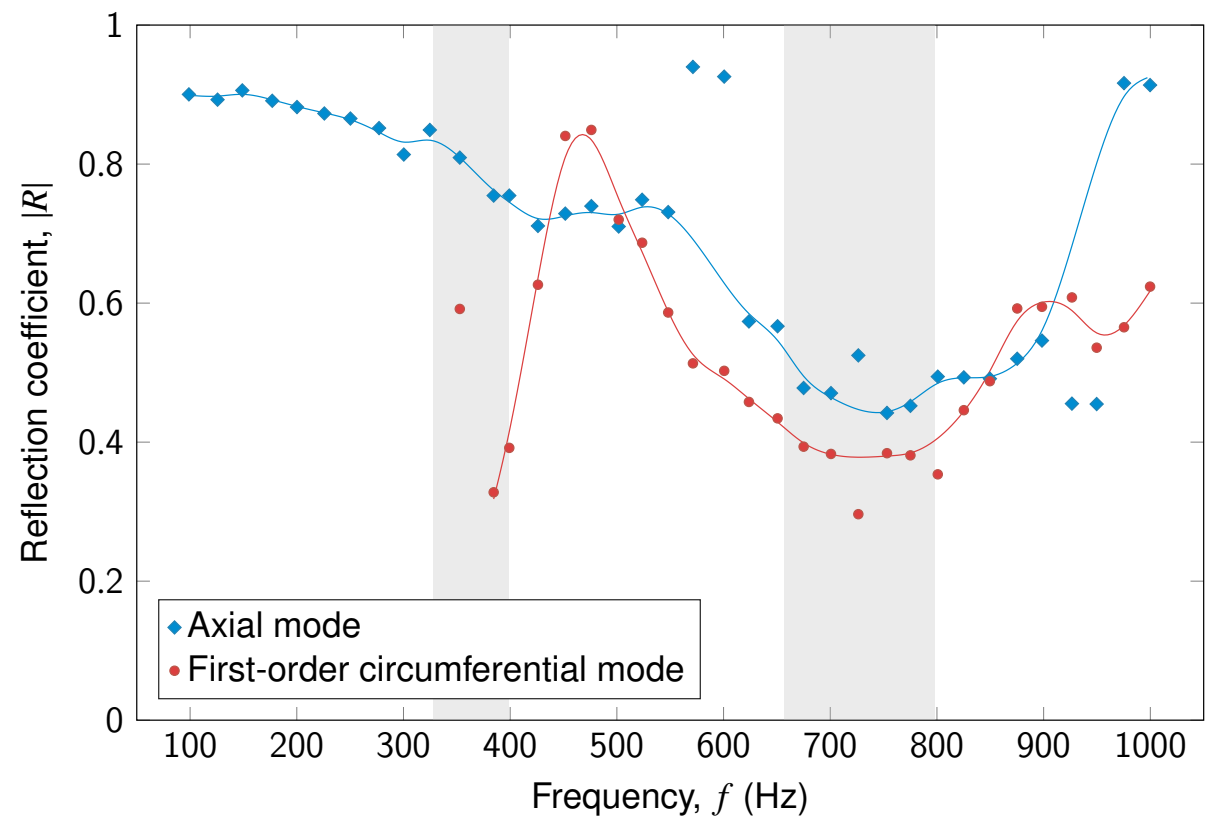

(a)

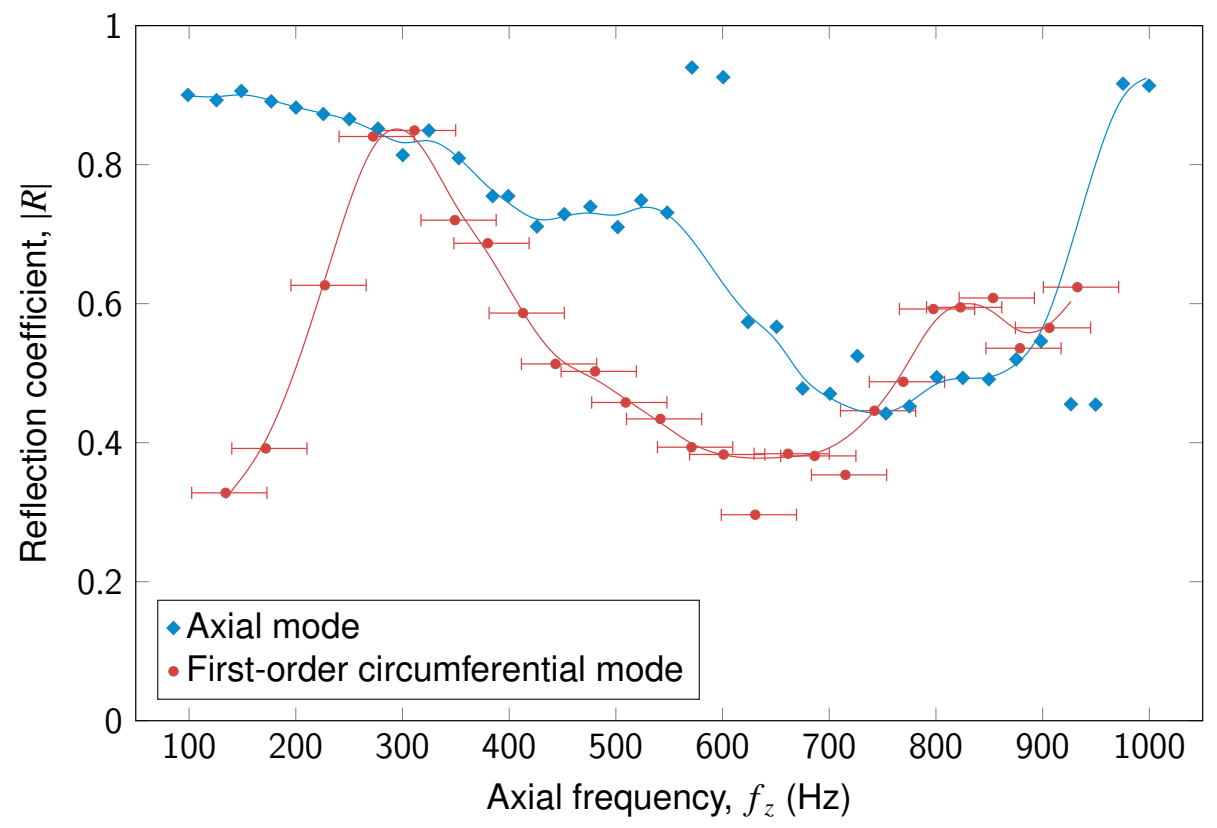

(b)

Figure 17: Reflection coefficient for the axial and first-order circumferential modes for the $22.5^{\circ}$ configuration and a dominant axial mode $\left(\Delta \bar{p} / p_{0}=3 \%\right)$ with: (a) the total frequency, and (b) the axial frequency component. 


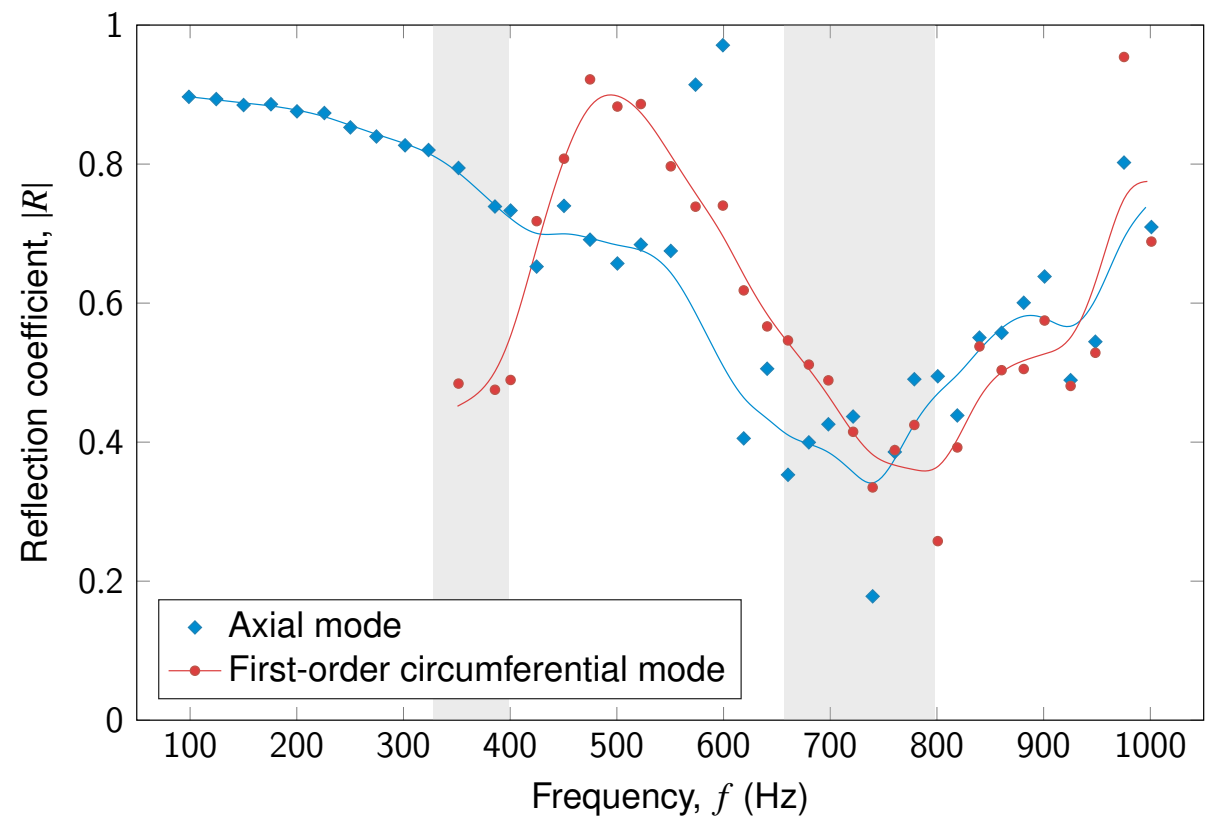

(a)

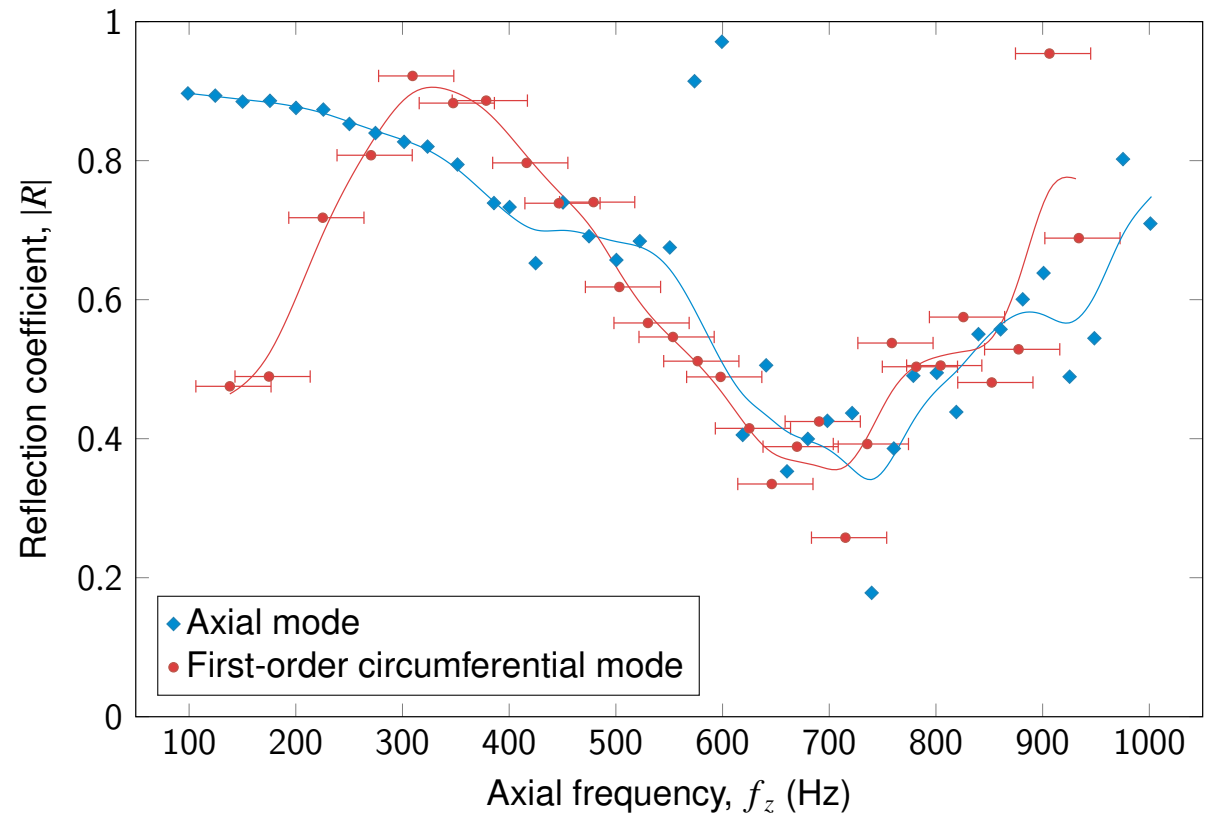

(b)

Figure 18: Reflection coefficient for the axial and first-order circumferential modes for the annular cavity and a dominant axial mode $\left(\Delta \bar{p} / p_{0}=3 \%\right)$ with: (a) the total frequency and (b) the axial frequency component. 


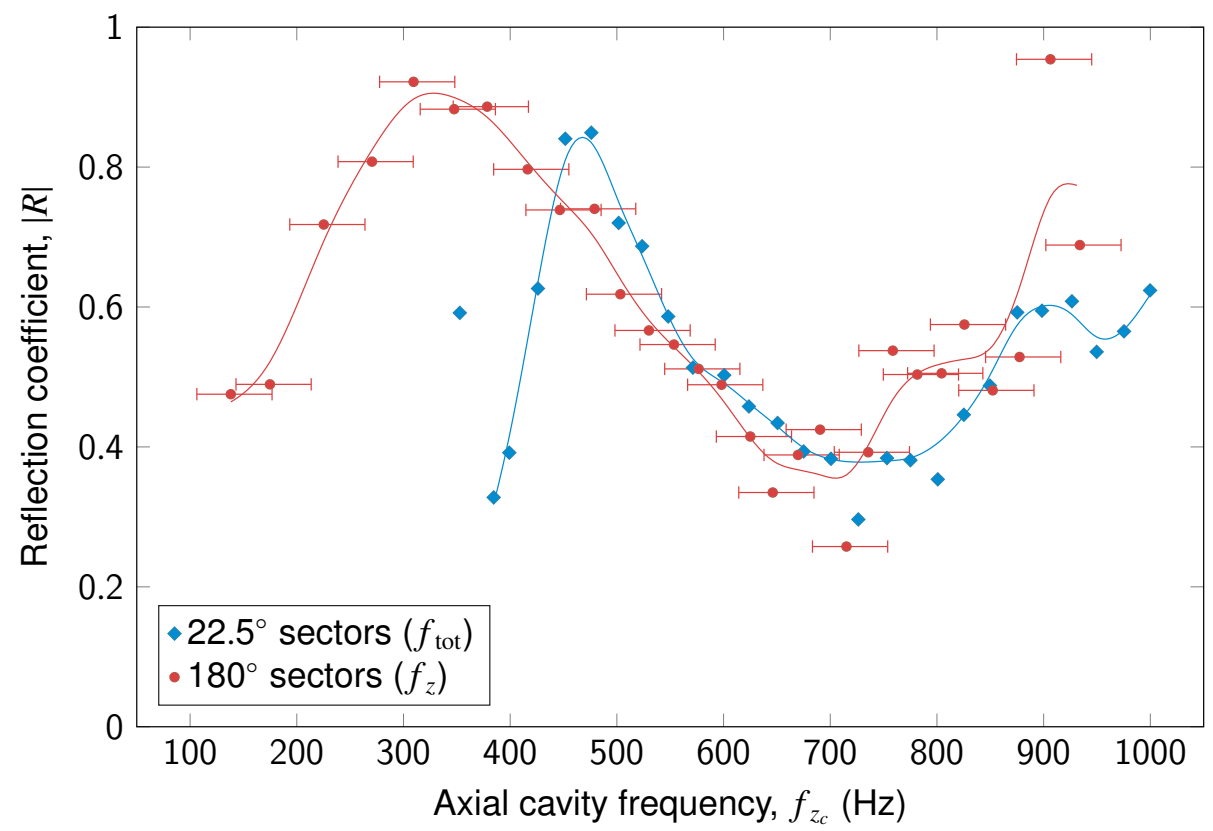

Figure 19: Reflection coefficient for the first-order circumferential modes with $22.5^{\circ}$ sectors and an annular cavity plotted against the axial cavity frequency $\left(\Delta \bar{p} / p_{0}=3 \%\right)$.

configurations. The helical wave model assumes that the field cuts-on uniformly based on the mean radius, although it can be modified to cut-on at the inner or outer wall radii. However, it fails to correctly capture, in all instances, the initial increase in the reflection coefficient from a value of approximately 0.5 at the mean radius cut-on frequency to a value of approaching one once the entire field has cut-on $(\approx 475 \mathrm{~Hz})$. The helical wave model accurately captures the response of the $180,360^{\circ}$, and annular configurations below $\sim 700 \mathrm{~Hz}$. However, above this there is some disparity observed, which could be related to the cut-on of the second-order circumferential mode and the potential transfer of energy to this mode. Thus, additional work is required to understand this characteristic with additional instrumentation to accurately decompose this mode simultaneously.

\section{Conclusions}

This work has shown the variation in performance of a perforated double-skin liner with a mixed acoustic mode field within a narrow annular duct. The damping system is mounted on the end of the duct at the axial termination so any axial plane waves present are uniformly incident upon the damper face. The liner is configured such that, based on the relative cavity volume per aperture on the 


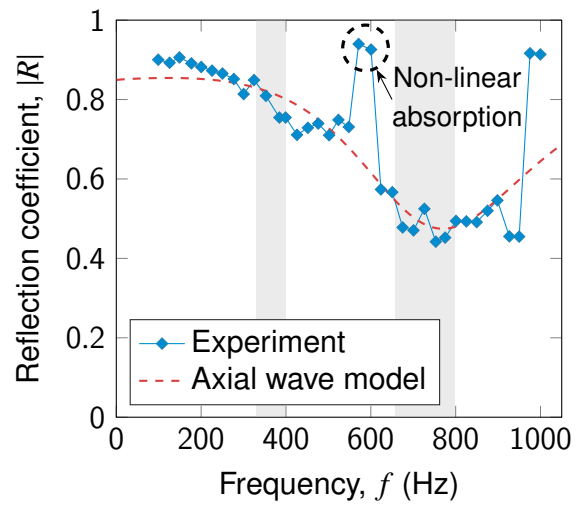

(a)

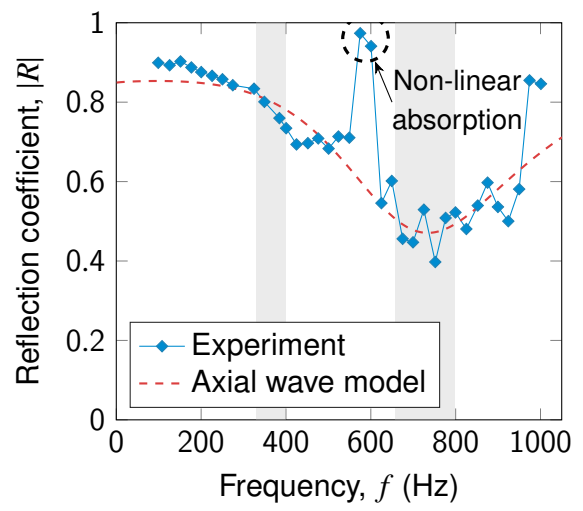

(c)

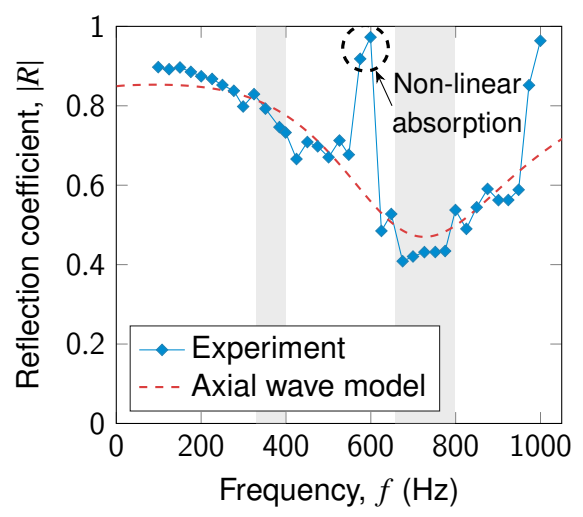

(e)

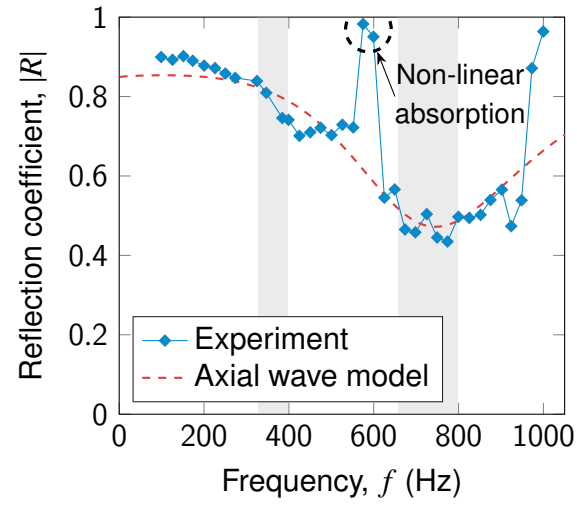

(b)

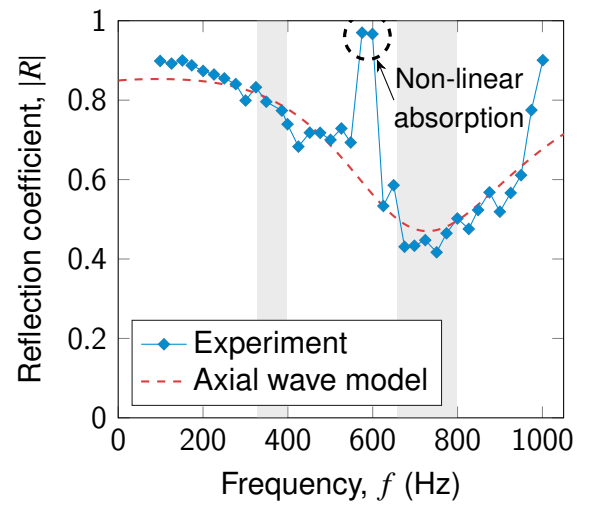

(d)

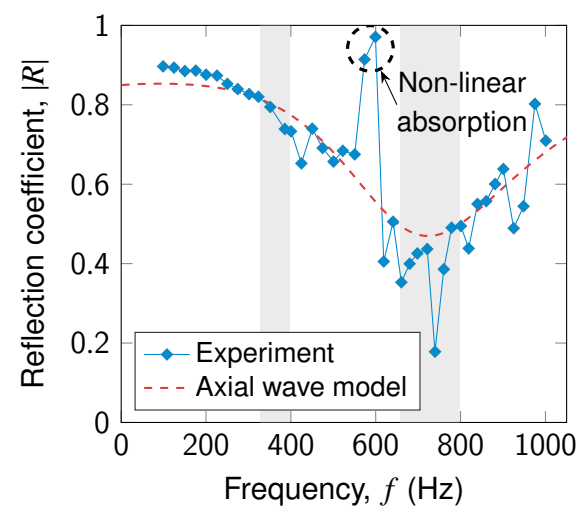

(f)

Figure 20: Comparison of the theoretical axial wave model and decomposed axial mode experimental data $\left(\Delta \bar{p} / p_{0}=3 \%\right.$ ). (a) $22.5^{\circ}$ sectors, (b) $45^{\circ}$ sectors, (c) $90^{\circ}$ sectors, (d) $180^{\circ}$ sectors, (e) $360^{\circ}$ sector, and (f) annular cavity. The grey shaded regions indicate the possible frequency ranges of first- and second-order circumferential mode cut-on. 


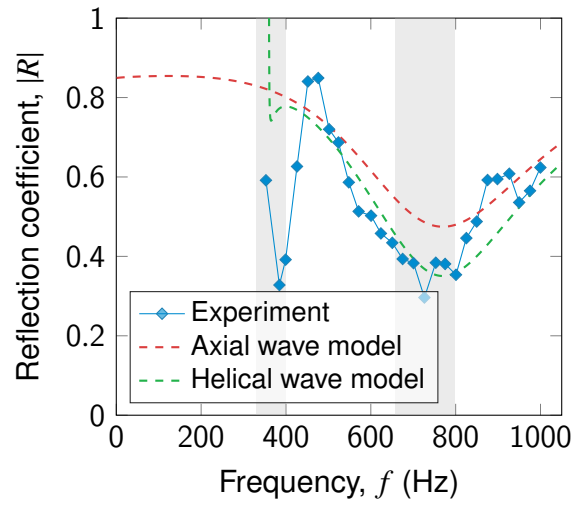

(a)

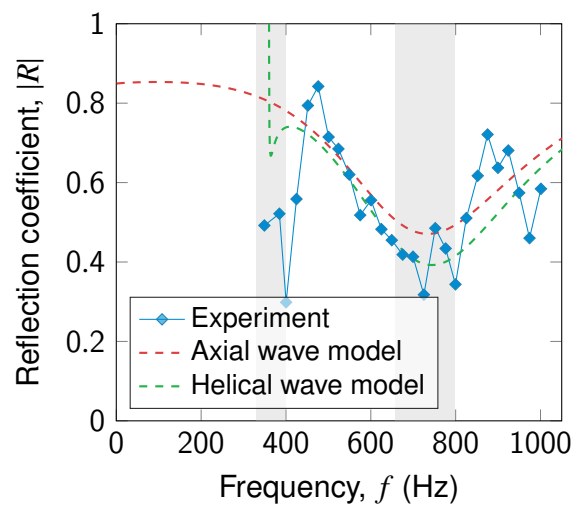

(c)

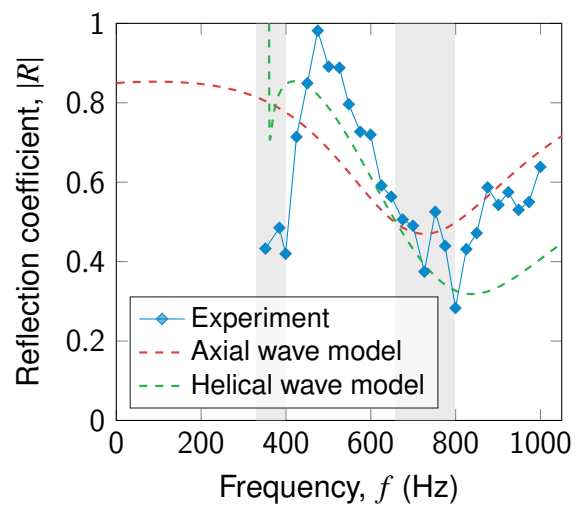

(e)

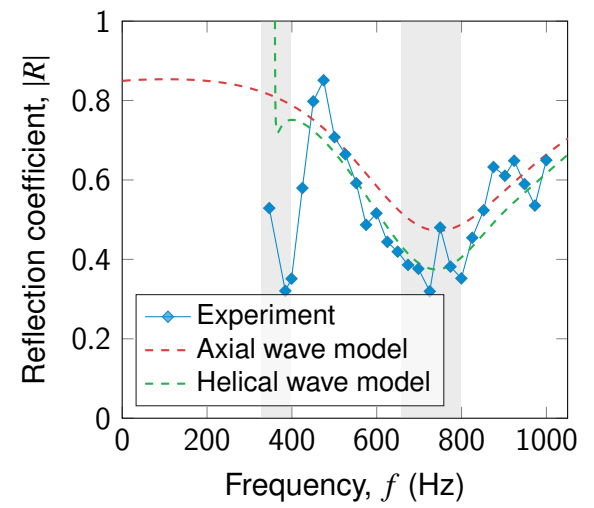

(b)

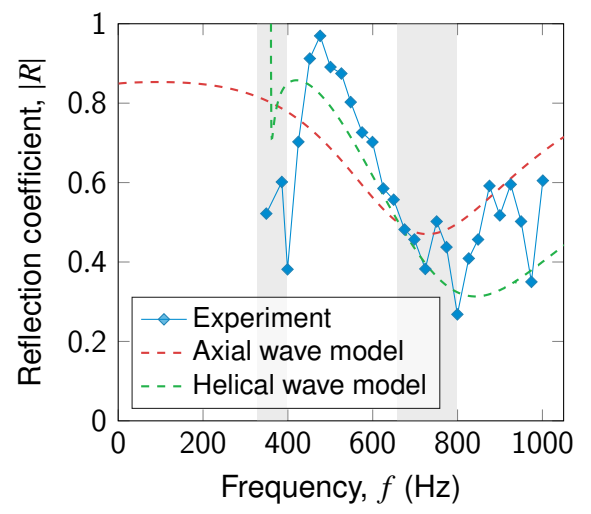

(d)

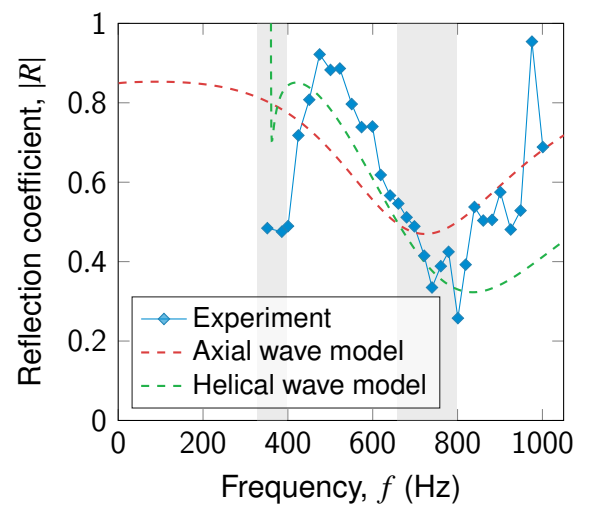

(f)

Figure 21: Comparison of the theoretical models and first-order circumferential mode experimental data for various liner cavity segmentations $\left(\Delta \bar{p} / p_{0}=3 \%\right.$ ). (a) $22.5^{\circ}$ sectors, (b) $45^{\circ}$ sectors, (c) $90^{\circ}$ sectors, (d) $180^{\circ}$ sectors, (e) $360^{\circ}$ sector, and (f) annular cavity. The grey shaded regions indicate the possible frequency ranges of first- and second-order circumferential mode cut-on. 
primary (damping) skin, it can resonate. Removal of circumferential segmentations within the cavity allow the potential generation of circumferential mode shapes that can alter the modal response. In this work, a quasi-one-dimensional model has been developed. The model captures this effect of circumferential mode generation within the cavity with an incident helical wave when the length of the rear cavity is significant relative to the wavelength of the circumferential component. Cavity resonance occurs for all the incident modes analysed, with the effect of circumferential mode generation causing the frequency at which resonance occurs to increase based on the circumferential frequency component.

An experimental facility was used to evaluate the damper performance where it was subjected to mixed acoustic modes with different orders simultaneously. The facility uses two loudspeaker inputs that are positioned around the circumference of an annular duct, which thus allows circumferential modes to cut-on. The amplitude and relative phase of the speakers can be controlled to dictate the dominant mode within the test facility. Analysis techniques have been developed to enable the decomposition and measurement of the mixed acoustic field. Experimental results, obtained from this facility, have been presented as an example data set that show both the impact of varying the total liner pressure drop and the effect of segmenting the cavity, with an incident dominant axial mode. The total acoustic response can be decomposed into the axial and circumferential modes to highlight the contribution of each mode. The same decomposition method has also been successfully applied to others in the data set across a range of axial and circumferential component combinations.

While the no mean flow case, causing non-linear absorption, has a large effect on the axial mode the change in the first-order circumferential mode for the geometry tested is relatively small. For the axial mode, it causes a narrowband response with high peak absorption and is thus suited for targeting a single specific frequency. As the liner pressure drop is increased this response becomes more broadband with reducing peak absorption, and as such targets a wider range of frequencies. While the same trends are observed for the first-order circumferential mode the effects are relatively small. However, in the non-linear absorption regime the absorption is a function of the incident acoustic pressure, with diminishing returns as the pressure increases. Non-linear absorption is observed at some frequencies even in the presence of flow due to the interaction of the acoustic pressure fields; however, these non-linear absorption effects are only noticeable within the axial mode. While the circumferential mode may provide non-linear absorption over some circumferential sections, there are simultaneously also areas of linear absorption, and thus from total damper response this non-linearity is less 
observable.

Segmenting the cavity, as shown, can potentially cause significant variations in the acoustic characteristics of the damper. The response of the axial mode is relatively unchanged by cavity segmentations, aside from a small frequency change caused by the volume difference. In the current experiment, this frequency variation was too small to measure accurately. The response of the first-order circumferential mode shows that for a cavity with a small angular length relative to the circumferential wavelength the damper can be considered to cause peak absorption at a similar frequency to the axial mode. This is shown experimentally to be true for the $22.5,45$, and $90^{\circ}$ configurations. However, a circumferentially large, or annular, sector causes the generation of mode shapes within the cavity that result in an increase in the frequency of peak absorption. The data can be shown to broadly collapse by calculating the axial frequency component of the wave; thus, proving that the increase is related to the circumferential cuton frequency. The cut-on frequency is, however, not well-defined and there is significant variation through the annulus based on the inner and outer diameters. This cut-on frequency disparity results in an initial increase in the reflection coefficient measured as the mode becomes cut-on throughout the annulus.

The analytical models generally have good agreement with the experimental data. The axial mode is well predicted by the axial plane wave model, which has been previously validated for other geometries on an experimental rig that geometrically limits the incident wave to an axial plane wave. Good agreement is achieved with the acoustically compact configurations for the circumferential mode. When a mode shape is generated within the cavity, the response below the cut-on of the second-order mode is good, although the rolling over of the reflection coefficient shortly after cut-on of the field is not captured correctly. The second-order mode cut-on causes disparity against the model that also requires further study.

\section{Acknowledgements}

This work has been financially supported by a Rolls-Royce plc and UK Engineering and Physical Sciences Research Council (EPSRC) industrial CASE studentship, which is gratefully acknowledged by the authors.

\section{References}

[1] J. D. Eldredge, A. P. Dowling, The absorption of axial acoustic waves by a perforated liner with bias flow, J. Fluid Mech. 485 (2003) 307-335. 
[2] J. Rupp, J. F. Carrotte, M. A. Macquisten, The use of perforated damping liners in aero gas turbine combustion systems, J. Eng. Gas Turbines Power 134 (2012) 071502.

[3] J. Rupp, Acoustic absorption and the unsteady flow associated with circular apertures in a gas turbine environment, Phd thesis, Department of Aeronautical and Automotive Engineering, Loughborough University, 2013.

[4] C. Jayatunga, Q. Qin, V. Sanderson, P. Rubini, D. You, W. Krebs, Absorption of normalincidence acoustic waves by double perforated liners of industrial gas turbine combustors, in: ASME Turbo Expo 2012, volume 2, ASME, Copenhagen, Denmark, 2012, pp. 611-621.

[5] M. A. Macquisten, A. Holt, M. Whiteman, A. J. Moran, J. Rupp, Passive damper LP tests for controlling combustion instability, in: ASME Turbo Expo 2006, volume 1, ASME, Barcelona, Spain, 2006, pp. 629-637.

[6] J. D. Eldredge, On the interaction of higher duct modes with a perforated liner system with bias flow, J. Fluid Mech. 510 (2004) 303-331.

[7] M. Bauerheim, F. Nicoud, T. Poinsot, Progress in analytical methods to predict and control azimuthal combustion instability modes in annular chambers, Phys. Fluids 28 (2016).

[8] P. Wolf, G. Staffelbach, A. Roux, L. Y. M. Gicquel, T. Poinsot, V. Moureau, Massively parallel LES of azimuthal thermo-acoustic instabilities in annular gas turbines, Comptes Rendus Mécanique 337 (2009) 385-394.

[9] P. Wolf, G. Staffelbach, R. Balakrishnan, A. Roux, T. Poinsot, Azimuthal instabilities in annular combustion chambers, Center for Turbulence Research, Proceedings of the 2010 Summer Program (2010) 259-269.

[10] S. Mendez, J. D. Eldredge, Acoustic modeling of perforated plates with bias flow for large-eddy simulations, J. Comput. Phys. 228 (2009) 4757-4772.

[11] S. Evesque, W. Polifke, Low-order acoustic modelling for annular combustors: validation and inclusion of modal coupling, in: ASME Turbo Expo 2002, volume 1, ASME, Amsterdam, The Netherlands, 2002, pp. 321-331.

[12] T. L. Parrott, W. R. Watson, Comparison of measured and calculated mode redistribution associated with spinning mode transmission through circumferentially segmented lined ducts, Technical Report NASA TM-84576, NASA Langley Research Center, Hampton, Virginia, 1983.

[13] E. Rademaker, S. Sarin, C. Parente, Experimental investigation on the influence of liner non-uniformities on prevailing modes, in: Aeroacoustics Conference, American Institute of Aeronautics and Astronautics, Reston, Viriginia, 1996.

[14] C. H. Gerhold, R. Cabell, M. C. Brown, Development of an experimental rig for investigation of higher order modes in ducts, in: 12th AIAA/CEAS Aeroacoustics Conference (27th AIAA Aeroacoustics Conference), American Institute of Aeronautics and Astronautics, Cambridge, Massachusetts, 2006.

[15] C. H. Gerhold, R. Cabell, D. E. Brown, Generation of higher order modes in a rectangular duct, in: ACTIVE 04: 2004 International Symposium on Active Control of Sound and Vibration, NASA Langley Research Center, NASA, Williamsburg, Virginia, 2004.

[16] W. R. Watson, Noise suppression characteristics of peripherally segmented duct liners, Technical Report NASA TP-1904, NASA Langley Research Center, Hampton, Virginia, 1981.

[17] W. R. Watson, Circumferentially segmented duct lines optimized for axisymmetric and standing wave sources, Technical Report NASA TP-2075, NASA Langley Research Center, Hampton, Virginia, 1982. 
[18] W. Bi, V. Pagneux, Mode propagation and attenuation in lined ducts, arXiv preprint arXiv:1404.1612 (2014).

[19] L. M. B. C. Campos, J. M. G. S. Oliveira, On the optimization of non-uniform acoustic liners on annular nozzles, J. Sound Vib. 275 (2004) 557-576.

[20] B. J. Tester, N. Baker, A. Kempton, M. Wright, Validation of an analytical model for scattering by intake liner splices, in: 10th AIAA/CEAS Aeroacoustics Conference, American Institute of Aeronautics and Astronautics, Reston, Viriginia, 2004, p. 2906.

[21] X. Liu, H. Jiang, X. Huang, S. Chen, Theoretical model of scattering from flow ducts with semi-infinite axial liner splices, J. Fluid Mech. 786 (2016) 62-83.

[22] W. Bi, V. Pagneux, D. Lafarge, Y. Aurégan, Modelling of sound propagation in a nonuniform lined duct using a Multi-Modal Propagation Method, J. Sound Vib. 289 (2006) 1091-1111.

[23] B. Regan, J. Eaton, Modelling the influence of acoustic liner non-uniformities on duct modes, J. Sound Vib. 219 (1999) 859-879.

[24] C. K. W. Tam, H. Ju, Finite difference computation of acoustic scattering by small surface inhomogeneities and discontinuities, J. Comput. Phys. 228 (2009) 5917-5932.

[25] S. Schmitt, D. Nürnberger, V. Carstens, Evaluation of the principle of aerodynamic superposition in forced response calculations, in: K. C. Hall, R. E. Kielb, J. P. Thomas (Eds.), Unsteady aerodynamics, aeroacoustics and aeroelasticity of turbomachines, Springer Netherlands, Dordrecht, 2006, pp. 133-144.

[26] C. L. Morfey, Sound transmission and generation in ducts with flow, J. Sound Vib. 14 (1971) 37-55.

[27] V. Bellucci, P. Flohr, C. O. Paschereit, Numerical and experimental study of acoustic damping generated by perforated screens, AIAA Journal 42 (2004) 1543-1549.

[28] V. Bellucci, P. Flohr, C. O. Paschereit, F. Magni, On the use of Helmholtz resonators for damping acoustic pulsations in industrial gas turbines, J. Eng. Gas Turbines Power 126 (2004) 271-275.

[29] C. L. Morfey, Rotating pressure patterns in ducts: their generation and transmission, J. Sound Vib. 1 (1964) 60-87.

[30] M. A. Cassell, Development of acoustic enhanced damping configurations for gas turbine combustion systems, Phd thesis, 2017.

[31] S. R. Stow, A. P. Dowling, T. P. Hynes, Reflection of circumferential modes in a choked nozzle, J. Fluid Mech. 467 (2002) 215-239.

[32] D. Biron, F. Simon, Characterization of acoustic sources, Technical Report, Department of Aerodynamics and Energetics Modelling, Office National d'Etudes et de Recherches Aérospatiales, Toulouse, France, 2003.

[33] J. J. Keller, E. Zauner, On the use of Helmholtz resonators as sound attenuators, Zeitschrift für angewandte Mathematik und Physik ZAMP 46 (1995) 297-327.

[34] G. F. Pickett, T. G. Sofrin, R. A. Wells, Method of fan sound mode structure determination, Technical Report NASA CR-135293, Pratt \& Whitney Aircraft Group, East Hartford, Connecticut, 1977.

[35] G. F. Pickett, R. A. Wells, R. A. Love, Method of fan sound mode structure determination computer program user's manual: Microphone Location Program, Technical Report NASA CR-135294, Pratt \& Whitney Aircraft Group, East Hartford, Connecticut, 1977. 